\title{
Photoaddition Reactions of Silyl Ketene Acetals with Aromatic Carbonyl Compounds: A New Procedure for $\beta$-Hydroxyester Synthesis ${ }^{\dagger}$
}

\author{
Ung Chan Yoon, Moon Jung Kim, Jac Joon Moon, Sun Wha Oh, Hyun Jin Kim, and Patrick S. Mariano \\ Deparment of Chemistry and Chemisty Instinte for functional Laterials, \\ Pusan tational linivensin. Pusan 609-735, Norea \\ 'Deparment of Chemistry, Lniversity of vew' Hexico. .1buquerque, New 1/exico 87131, LS.1 \\ Receivedilpril 2. 2002
}

\begin{abstract}
Photochemical reactions of aromatic carbonyl compounds with silyl ketene acetals lave been explored. lrradiation of acetonitrile or benzene solutions containing aryl aldehydes or ketones in the presence of silyl ketene acetals is observed to promote formation of $\beta$-hydroxyester. 2.2-dioxyoxetane and 3,3-dioxyoxetane products. The ratios of these photoproducts, which arise by competitive single electron transfer (SET) and classical Patemo-Buchi mechanistic pathways, is found to be dependent on the degree of methyl-substitution on the viny 1 moieties of the ketene acetals in a manner which reflects expected alkyl substituent effects on the oxidation potentials of these electron rich donors. An analy sis of the product distribution arising by irradiation of a solution containing butyrophenone (6) and the silyl ketene acetal 9. derived from methyl isobuty rate. provides an estimate of the rate constants for the competitive Norrish type Il. SET and Patemo-Buclu processes occuring. Finally, sequences involving silyl ketene acetal-aryl aldehyde or ketone photoaddition followed by 2.2-dioxyoxetane lydrolysis represent useful procedures for Claisen-condensation type, $\beta$-hydroxyester sỵnthesis.
\end{abstract}

Key Words : Photoaddition reaction. Silyl kelenc acclals. Aromatic carbonyl compound. $\beta$-Hydroxyester. Single electron transfer photochemistry

\section{Introduction}

The operation of sequential single electron transfer (SET)desilylation pathways in the photochemistry of acceptor $/ \alpha$ silyl electron donor systems is now well-documented. ${ }^{1}$ Product formation in photochemical reactions which follow: this mechanistic course is typically highly selective owing to the fact that transfer of a silyl group from an $\alpha$-silyl cation radical intermediate to a silophile often occurs more rapidly than other possible $\alpha$-heterolytic fragmentation modes such as base induced deprotonation. - Consequently, sequential SET-desilylation can serve as an efficient and regioselective pathway leading to carbon-centered radical generation and. eventually: to carbon-carbon bond formation (Scheme 1). ${ }^{1-7}$ Examples of SET-promoted excited state processes in which intermediate cation radical desilylation serves as the driving force are found in the photochemistry of iminium salts. ${ }^{3}$ cyanoarenes. ${ }^{+}$conjugated-cyclohexenones. ${ }^{5}$ ortho-quinones ${ }^{4}$ and phthalimides.

Our interests in this area of photochemistry has encouraged a recent exploration probing the photochemical reactivity of aryl aldehyde and ketone/silyl ketene acetal systems. Consideration of the chemical and redox properties of excited states of aromatic carbonyl compounds and ground states of silyl ketene acetals suggests that these substrate

I']he authors dedicate this paper to the memory of J'ofessor Sang Chul shim who has been a great organic photochemist and a prominent leader of the rapidly advancing Korean chemical community:

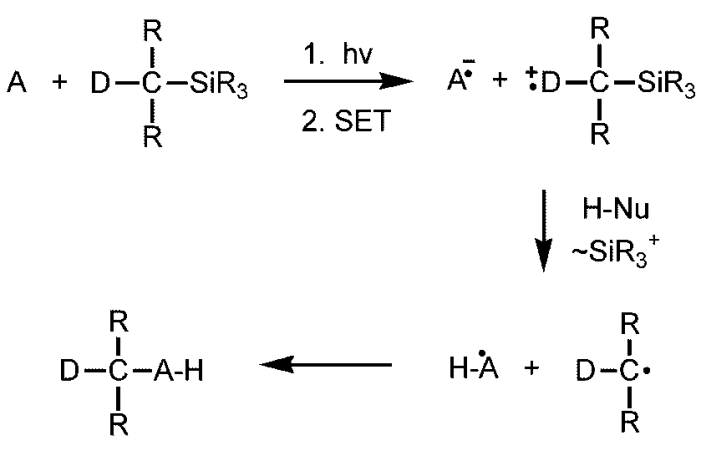

Scheme 1

combinations have the potential of participating in several types of photochenical reaction pathways. For. example. SET from the electron rich silyl ketene acetal donors to aryl aldehyde and ketone excited states should be thermodynamically favorable (see below) and. as a result. it might effectively compete with other excited state deactivation modes to generate ion radical interınediates I (Schenle 2). Collapse of these species to produce zwitterionic IV or radical III intermediates could serve to drive formation of the respective oxetane VI or $\beta$-hydroxyester $\mathbf{V}$ products. Alternatively, excited state reactivity of aryl aldelyde and ketone/silyl ketene acetal sytem might adhere to classical patterens in which case oxetanes II arising by Paterno-Buchi type cycloaddition $\$$ would be the major products.

The mechanistic questions posed above and their potential synthetic consequences have stimulated an exploration of the photoreactions occuring between a variety of aryl aldehydes 


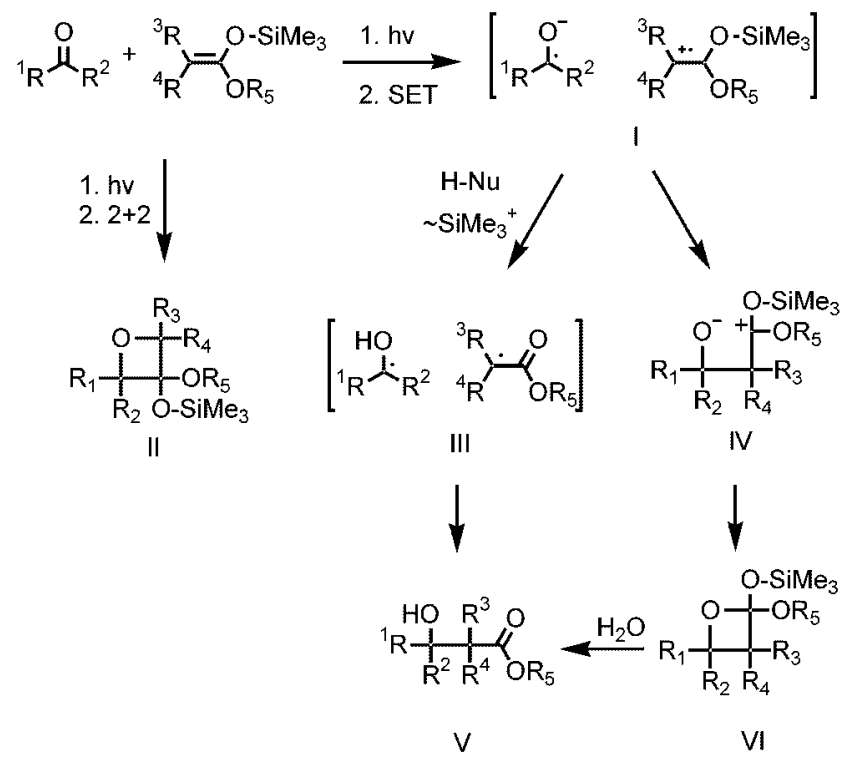

Scheme 2

and ketones 1-8 and silyl ketenc acctals 9-12. Below. we present the results of this study" which demonstratc that (l) aromatic carbonyl compounds and silyl ketenc acctals ${ }^{\text {li }}$ participate in two competing cxcilcd state reaction pathways involving SET-induced and classical oxctanc formation. the relative efliciencies of which are dependent upon the nature of the silyl ketene acetal donors. (2) SET-induced oxctanc formation competes with Norrish type II processes in the photochemistry of ary ketones which possess $\gamma$-hydrogens. and (3) oxetanes arising by the SET route and containing silyl ortho ester functionality are readily translormed to $\beta$ hydroxyesters. thus. providing an eflicient procedure for preparation of these Claisen-condensation lype products.

\section{Results}

Photochemical reactions were performed by irradiation of $\mathrm{CH}_{3} \mathrm{CN}$ or benzene solutions of the carbonyl compounds 1-8 $(30-37 \mathrm{mM})$ and the silyl ketene acetals 9-12 (37-72 $\mathrm{mM})$ by using Pyrex filtered-light $(\lambda>290 \mathrm{~mm})$ for time periods resulting in 26-100\% conversion of the carbonyl compounds. Product separation in each case was performed by silica gel chromatography. Irradiation times, solvents, products and yields for these processes are recorded in Table 1.

By viewing the data in Table 1, it can be seen that irradiation of the aromatic carbonyl compounds in solutions containing the silyl ketene acetals results in the predomimant formation of three kinds of products including 3-ary 1-3hydroxyesters V. 2-methosy-2-sily loxy-4-aryloxetanes VI and 2-ary 1-3-methoxy -3-sily loxyoxetanes II (see Scheme 2). In addition. the aromatic ketones 2.3 and 5 produce the dimers 47.48 and 49 by photoreduction while carbonyl compounds 1 and 5 generate 3-ary 1-3-sily loxyesters 42 and $\mathbf{4 3}$ as minor products. The ratios of the major products. II. $\mathbf{V}$ and VI. are observed to be dependent on the nature of the silyl ketene acetal substrates 9-12. Specifically. this is seen in the effect of alkyl substitution in the silyl ketene acctal on the relative amounts of 2-ary 1-3-methoxy-3-sily loxyoxetane II w. 3-ary 1-3-hydroxyesters $V$ and 2-methoxy-2-sily loxy -taryloxetanes VI that are produced in these photochemical processes. Thus, formation of 3-aryl-3-hydroxy esters and their precursors. the 2-methoxy-2-silyloxy-4-ary loxctanes (sce below), greatly predominates over that of the 2-ary 1-3-

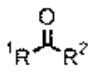

$$
\begin{aligned}
& 1\left(R^{1}=P h, R^{2}=H\right) \\
& 2\left(R^{1}=P h, R^{2}=M e\right) \\
& 3\left(R^{1}=P h, R^{2}=P h\right) \\
& 4\left(R^{1}=p--M e O-P h . R^{2}=M e\right) \\
& 5\left(R^{1}=p-C N-P h, R^{2}=M e\right) \\
& 6\left(R^{1}=P h, R^{2}=n-P r\right) \\
& 7\left(R^{1}=P h, R^{2}=n-B u\right) \\
& 8\left(R^{1}=2-n a p h+h y l, R^{2}=M e\right)
\end{aligned}
$$

$$
\text { OMe }
$$

$13\left(R^{1}=P M ; R^{2}=H, R^{3}=R^{4}=M e\right)$ $14\left(R^{1}=P h . R^{2}=R^{3}=R^{4}=M e\right)$ $15\left(R^{1}=R^{2}=P h, R^{3}=R^{4}=M e\right\}$ $16\left(R^{1}=p-M e O-P h . R^{2}=R^{3}=R^{4}=M e\right)$ $17\left(R^{1}=p-C N-P h . R^{2}=R^{3}=R^{4} \approx M e\right)$ $18\left(R^{1}=\mathrm{Ph}_{2} \mathrm{R}^{\hat{k}}=\mathrm{n}-\mathrm{Pr}, \mathrm{R}^{3}=\mathrm{R}^{4}=\mathrm{Me}\right)$ $19 R^{1}=2$-naphithyl. $R^{2}=R^{3}=R^{4}=$ Mej $20\left(R^{1}=P h, R^{2}=R^{3}=M e, R^{4}=H\right\}$ $21\left\{R^{1}=R^{2}=P h, R^{3}=M e, R^{4}=H\right\}$ $22\left(R^{1}=\right.$ p-MeO-Ph, $\left.R^{2}=R^{3}=M e, R^{4}=H\right)$ $23\left(R^{1}=p-C N-P h . R^{\prime \prime}=R^{3}=M e . R^{4}=H\right)$ $24\left(R^{1}=\mathrm{Ph} . R^{2}=\mathrm{me}: R^{3}=R^{4}=H\right.$ H) $25\left(R^{1}=R^{2}=P h, R^{3}=R^{4}=H i\right.$ $26\left(R^{1}=\mathrm{p}-\mathrm{CN}-\mathrm{Ph}, \mathrm{R}^{\prime \prime}=\mathrm{M}, \mathrm{R}^{3}=\mathrm{R}^{4}=\mathrm{H}\right)$

$$
\begin{gathered}
42\left(R^{1}=P h \quad R^{2}=H\right) \\
43\left(R^{1}=P-C N \cdot P h . R^{2}=M e\right) \\
47\left(R^{1}=P h . R^{2}=M e\right) \\
48\left(R^{1}=R^{2}=P h\right) \\
49\left(R^{1}=P-C N-P h . R^{2}=M e\right)
\end{gathered}
$$

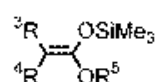

$9\left(R^{3}=R^{4}=R^{5}=M e\right)$ $10\left(R^{3}=R^{5}=M e, R^{4}=H\right.$ $11\left(R^{3}=R^{4}=H, R^{5}=M e\right)$ $12\left(R^{3}=\mathrm{H}, \mathrm{F}^{4} \cdot \mathrm{R}^{\mathrm{f}-1}=\mathrm{CH}_{2} \mathrm{CH}_{2} \mathrm{CH}_{2}-\right)$

$$
\underbrace{\mathrm{O}-\mathrm{R}^{4}}_{\mathrm{R}^{2} \mathrm{R}^{3}}
$$

$27\left\langle R^{1}=P h, R^{3}=H, R^{3}=R^{2}=M e\right\}$ $28\left(R^{1}=P h, R^{2}=R^{3}=R^{4}=M e\right)$ $29\left\{R^{1}=R^{2}=P h \cdot R^{3}=R^{4}=M e\right\}$ 30 ( $R^{1}=P h, R^{2}=n-P r \quad R^{3}=R^{4}=M e$ )

$$
\underbrace{\mathrm{CSiMe}_{3}}_{\mathrm{R}^{2} \mathrm{OCH}_{3}}
$$

$31\left(R^{1}=P h, R^{2}=H \cdot R^{3}=R^{4}=M e\right)$ $32\left(R^{1}=P h, R^{2}=R^{3}=R^{4}=M e\right)$ $33\left(R^{1}=R^{2}=P h, R^{3}=R^{4}=M e\right)$ $34\left(R^{1}=P h, R^{2}=R^{3}=\right.$ Me $\left.R^{4}=H\right)$ $35\left(R^{4}=\right.$ p-MEO-Ph, $R^{2}=R^{3}=$ Ae $\left.: R^{4}=H\right)$ $36\left(R^{7}=\rho-C N-P h . R^{2}=R^{3}=\right.$ Me, $\left.R^{4}=H\right)$ $37\left(R^{1}=R^{7}=\right.$ Fh. $R^{3}=$ Mes. $\left.R^{4}=H\right)$ $38\left(R^{1}=P h, R^{2}=M e, R^{3}=R^{4}=H\right)$ $39\left(R^{1}=R^{2}=P+R_{1} R^{3}=R^{4}=H\right)$ $40\left(R^{\top}=p-C N-P h, R^{2}=M e, R^{3}=R^{4}=H\right)$ $44\left(R^{i}=P h, R^{2}=n-P r, R^{3}=R^{4}=M E\right)$

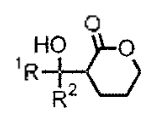

$44\left\langle R^{-}=P h, R^{2}=\right.$ Me $\rangle$ $45\left\{R^{\prime}=R^{2}=P h\right\}$ $46\left(R^{4}=p-C N w P h, R^{2}=M e\right)$

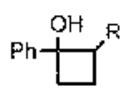

So $(\mathrm{R}=\mathrm{H})$ methoxy-3-sily losyoxetanes in the photoreactions of the dimetly1-substituted ketene acetal 9 . In contrast, reactions of carbonyl compounds with the non-metlyl substituted silyl ketene acetal 11 lead to the predominant production of 2ary l-3-methoxy-3-silyloxyoxetanes (entries 19-23, 31) while photoreactions with mono-methyl ketene acetal 10 generate both 3-aryl-3-hydroxy esters and 2-anyl-3-methoxy-3-silyoxyoxetanes in roughly equal ratios (entries 12-18). Finally. the cyclic ketene acetal $\mathbf{1 2}$ produces Claisen-type products 
44-46 exclusively.

It is interesting that the 2-methoxy-2-sily loxy-4-aryloxetane products VI. which contain labile ortho ester functions. are observed to undergo rapid hydrolysis to form the Claisen-condensation type $\beta$-hydroxyesters products $\mathbf{V}$. As a result of this property. 2-methoxy-2-sily loxy-4-aryloxetanes are isolated as major products only in the reactions of carbony 1 compounds 1-3 with only the dimethyl-substituted silyl ketene accial 9. In the other processes. only $\beta$ hydroxyesters are isolated and these derive from hydrolysis of the more labile 2.2-dioxyoxetanes during work-up and chromatographic separation.

Photoreactions of butyrophenone (6) with the dimethyl silyl ketene acctal $9(68 \mathrm{mM})$ in $\mathrm{CH}_{3} \mathrm{CN}$ and benzene (entrics 27 and 28) produce the Norrish type ll products acctophenone (2. 45\% and 31\%) and l-pheny lcyclobutanol (50). $4 \%$ and $6 \%$ ) along with the adducts. 3-pheny 1-3-propy l3-hydroxyester (18). 2-methoxy-2-sily loxy-4-phenyl-4-propyloxetanc (30) and 3-methoxy-3-siloxy-2-pheny i-2-prop- yloxetane (41). Howcver photoreactions of valcrophenone (7) in the presence of 9 are dominated by products arising from Norrish type II pathways (2 and 51) and sccondary photorcaction of 2 with 9 (i.e. 14 and 32).

\section{Discussion}

Two competing mechanistic pathways appear to bc responsible for formation of the major 3-ary 1-3-hydroxyester V. 2-methoxy-2-sily loxy-4-ary loxelane VI and 2-ary 1-3-methoxy-3-sily loxyoxetane II (sec Scheme 2) products produced in the photorcactions of an 1 aldehydes and ketones with silyl ketene acctals. Excitation of the aromatic carbonyl 3sily loxyoxetane II (sec Scheme 2) products produced in the photorcactions of aryl aldehydes and ketones with silyl ketenc acctals. Excitation of the aromatic carbonyl compounds in cach case is followed by rapid intersy stem crossing $\left(k_{150}=\right.$ ca. $1 \times 10^{16} \mathrm{~s}^{-1}$ ) to produce the corresponding triplet excited states which have long lifetimes $(0.15-12 \mathrm{~ms}))^{\text {" }}$ Elcctron

Table 1. Photoreactions of Aryl Kelones 1-8 and Silỵl Ketene Aectals 9-12"

\begin{tabular}{|c|c|c|c|c|c|}
\hline Lintry & Reactants & Solvent & $\begin{array}{c}\text { Reaction time } \\
\text { (l) }\end{array}$ & $\begin{array}{c}\text { Conversion } \\
(\%)\end{array}$ & Products $(\% \text { yick })^{\prime}$ \\
\hline 1 & $1+9$ & $\mathrm{CI} \mathrm{H}_{3} \mathrm{CN}$ & 3 & 98 & $13(76), 30(5)$ \\
\hline 2 & $1+9$ & Benzenc & 11 & 79 & $13(72), 31(8), 27(4), 42(14)$ \\
\hline 3 & $2+9$ & $\mathrm{CH} \mathrm{H}_{3} \mathrm{CN}$ & 16 & 100 & $28(83), 32 \mathrm{a}(5)+32 \mathrm{~b}(6), 47(5)$ \\
\hline 4 & $2+9$ & Benzenc & 33 & 77 & $28(64), 32 a(11), 32 b(7), 47(3)$ \\
\hline 5 & $3+9$ & $\mathrm{CH} \mathrm{H}_{3} \mathrm{CN}$ & 6 & 100 & $15(13), 29(65), 48(23)$ \\
\hline 6 & $3+9$ & Benzens & 4 & 95 & $15(11), 29(27), 33(3), 48(40)$ \\
\hline 7 & $4+9$ & $\mathrm{CH}, \mathrm{CN}$ & 43 & 74 & $16(62)$ \\
\hline 8 & $5+9$ & $\mathrm{CH} \mathrm{H}_{3} \mathrm{CN}$ & 28 & 88 & $17(32)+43(10), 49(34)$ \\
\hline 9 & $5+9$ & Benzenc & 10 & 86 & $17(86), 43$ (trace $), 49(5)$ \\
\hline 10) & $5+9$ & $35 \% \mathrm{II}_{2} \mathrm{O}-\mathrm{CI}_{3} \mathrm{CN}$ & 37 & 62 & $17(11)+43(3), 49(72)$ \\
\hline 11 & $8+9$ & $\mathrm{CI} H_{3} \mathrm{CN}$ & 40 & 44 & $19(11)$ \\
\hline 12 & $2+10$ & $\mathrm{CI} \mathrm{H}_{3} \mathrm{CN}$ & 12 & 72 & $20 a(19), 20 b(24), 34 a(8), 34 b(16), 47(16)$ \\
\hline 13 & $2+10$ & Benzenc & 14 & 47 & $20 \mathrm{a}(15), 20 \mathrm{~b}(25), 34 \mathrm{a}(21), 34 \mathrm{~b}(34)$ \\
\hline 14 & $3+10$ & $\mathrm{CI} \mathrm{H}_{3} \mathrm{C} \mathrm{N}$ & 8 & 85 & $21(41), 37 a(12), 37 b(25), 48(6)$ \\
\hline 15 & $3+10$ & Benzenc & 7 & 90 & $21(39), 37 a(12), 37 b(24), 48(14)$ \\
\hline 16 & $4+10$ & CIH CN & 50 & 26 & $22 a(17), 22 b(18), 35 a(26), 35 b(22)$ \\
\hline 17 & $4+10$ & Benzene & 19 & 46 & $22 a(24), 22 b(31), 35 a(18), 35 b(25)$ \\
\hline 18 & $5+10$ & Benzenc & 13.5 & 87 & $23 a(17), 23 b(47), 36 a(8), 36 b(17), 49(5)$ \\
\hline 19 & $2+11$ & $\mathrm{CH} \mathrm{CH}_{3} \mathrm{~N}$ & 20 & 49 & $24(25), 38 a(16), 38 b(30)$ \\
\hline 20) & $2+11$ & Benzenc & 16 & 65 & $24(22), 38 a(21), 38 b(32)$ \\
\hline 21 & $3+11$ & Benzenc & 25 & 89 & $25(16), 39(68), 48(4)$ \\
\hline 22 & $4+11$ & Benzene & 48 & 0 & no reaction \\
\hline 23 & $5+11$ & Benzene & 25 & 43 & $26(40), 40 a(1+4)+40 b(32)+49(6)$ \\
\hline 24 & $2+12$ & $\mathrm{CI} \mathrm{I}_{i} \mathrm{CN}$ & 37 & 30 & $\mathbf{H}(21)$ \\
\hline 25 & $3+12$ & $\mathrm{CH} \mathrm{H}_{3} \mathrm{CN}$ & 21 & 61 & $45(20), 48(20)$ \\
\hline 26 & $5+12$ & Benzenc & 25 & 54 & $46(20)+49(50)$ \\
\hline 27 & $6+9$ & $\mathrm{CH} \mathrm{H}_{3} \mathrm{CN}$ & 9 & 100 & $2(45), 18(29), 30(5), 28 a(3), 28 b(3),+1 a(3), 42 b(4) .50(4)$ \\
\hline 28 & $6+9$ & Benzenc & 8 & 95 & $2(31), 18(25)-30(7)-28 a(2) 28 b(3)+1 a(11)+4 b(3) .50(6)$ \\
\hline 29 & $7+9$ & $\mathrm{CH}_{3} \mathrm{CN}$ & 5.5 & 96 & $2(31), 14(14), 32 a(1), 32 b(2), 51 a(7), 51 b(19)$ \\
\hline 30 & $7+9$ & Benzene & 6.5 & 88 & $2(18) .14(11), 32 a(8), 32 b(13) .51 a(13) .51 b(19)$ \\
\hline 31 & $6+11$ & $\mathrm{CH}_{3} \mathrm{CN}$ & 2 & 95 & $2(63) .50(17)-38 a(3)-38 b(7)$ \\
\hline
\end{tabular}

"Concentrations of reactants. [hetone]: [acetal] are 3672 (mM) for cutriesl-2.3, 3060) (mM) tor entrics 24-26, 34 68 (mM) for entrics 27-28, 31, and 3737 (mM) for entries 25)-30. "Yields are based on consumed carbonyl compounds. 
transfer from the dimethy 1 -substituted sily 1 ketene acetal 9 $\left(E_{1: 2}(+)=+0.66 \mathrm{~V}\right)^{12}$ to the triplet excited ary 1 aldehy de or ketone $\left(\mathrm{E}^{\mathrm{T}}{ }_{1}(-)=-1.19 \sim-1.28 \mathrm{~V}\right)^{11.13}$ is thermodynamically favorable $\left(\Delta \mathrm{G}_{\mathrm{SET}}=c \alpha \cdot-0.5 \sim-0.6 \mathrm{eV}\right)$ and thus should occur rapidly ${ }^{14}$ for the accial concentrations in the range of $37-72$ $\mathrm{mM}$ used in these processes. As depicted in Scheme 2 above. the SET-process results in production of the ion radical pair $\mathbf{I}$ which can partition to the $\beta$-hydroxyester $\mathbf{V}$ or 2.2-dioxy oxetanes $\mathbf{V l}$ adducts by respective desilylation and zwittcrion forming $\mathrm{C}-\mathrm{C}$ bonding pathways. Owing to the lability of the ortho-ester containing oxetanes VI. they undergo rapid hydroly tic conversion to the $\beta$-hydroxyesters $V$ in all cases except those arising from the dimethylsubstituted ketene accial 9.

In competition with these routes. direct crcloaddition of the ketene acctals with the triplet carbonyl compounds occurs to generate classical Paterno-Buchi products. ${ }^{8}$ 3.3dioxyoxetanes II. The relative efliciencies of the SET and classical cycloaddition reactions should be governed by the oxidation potential of the silyl ketene acetal which. in turn. is a function of the degree of methyl substitution on the vinyl moicty. Thus. the predominant production of 2.2-dioxyoxetanes (from SET roules) in photorcactions with the dimethylsubstituted acctal 9 verstis 3.3-dioxyoxctanes (from PatcrnoBuchi routes) with the non-methyl-substuluted analog 11 clearly reflects the controll oflered by this property.

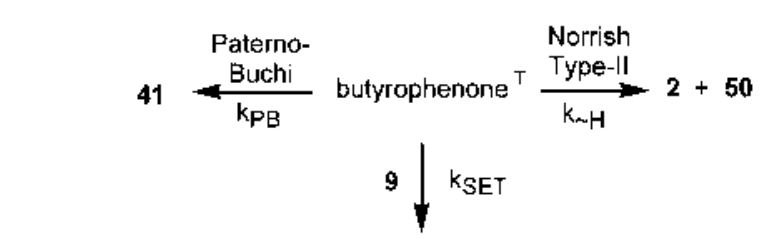

$\underset{+}{\text { butyrophenone }}+\stackrel{+}{9]}\left[\begin{array}{c}\text { butyrophenone } \\ {[9]^{+}}\end{array}\right] \underset{\mathrm{k}_{\text {oxet }}}{\longrightarrow} 18+28+30$

Scheme 3

Obscrvations made in studics with butyrophenone (6) provide qualitative inforntation about the kinetics of oxelane formation by the SET mechanistic pathway. The ratc constant for buty rophenone triplet $\gamma$-hydrogen abstraction (leading to 2 and 50 ) has been measured previously $\left(k_{\text {II }}=8 \times 10^{6} \mathrm{sec}^{-1}\right) !^{15}$ As shown in Scheme 3. this process competes with classical Paterno-Buchi reaction to produce oxetane $\mathbf{4 1}\left(k_{\mathrm{PD}} \times\right.$ [keten acetal]) and SET from the silyl ketene acetal 9 with a bimolecular rate that is controlled by $k_{\mathrm{SET}}\left(\alpha a .1 \times 10^{111} \mathrm{M}^{-1}\right.$ $\left.\mathrm{s}^{-1}\right)^{14}$ and the acetal concentration $\left(6.8 \times 10^{-2} \mathrm{M}\right)$. The ion radical pair produced following SET then partitions to ground state starting materials by back electron transfer $\left(k_{\mathrm{DSET}}=c \alpha .1 \times 10^{113} \mathrm{M}^{-1} \mathrm{~s}^{-1}\right)$ or to oxetanes $28+30$ and $\beta-$ hydroxyester 18 by sequential $\mathrm{C}-\mathrm{C}$ and $\mathrm{C}-\mathrm{O}$ bond formation ( $\left.k_{\text {oxet }}\right)$. The kinetic sequence depicted in Scheme 3 translates into the Norrish Type II to Paterno-Buchi and SET-oxetane photoreaction quantum efficiencies given in equations 1 and 2. Based upon these formulations. estimated rate constants given above and the product ratios. we estimate that in the reaction of ketcne acetal 9 with triplet buty rophenone $k_{\mathrm{PB}}=$ ca. $2 \times 10^{7} \mathrm{M}^{-1} \mathrm{~s}^{-1}$ and $k_{\text {oxet }}=c a .1 \times 10^{8} \mathrm{M}^{-1} \mathrm{~s}^{-1}$. Intercstingly. SET is not a competitive route followed in the photoreaction of valerophenone (7) with ketene acetal 9 . The major reason for this lies in the rate for intramolecular $\mathrm{H}$-atom abstraction in triplet valcrophenone $\left(1.3 \times 10^{8} \mathrm{sec}^{-1}\right)^{15}$ which is much larger than diffusion controlled bimolecular SET in the concentration range $\left(10^{-2} \mathrm{M}\right)$ of 9 used in these cxperiments.

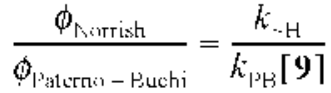

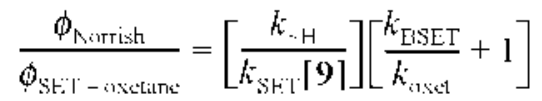

Finally. it is worth mentioning that photoreactions of aromatic carbonyl compounds with silyl ketene acetals in most cases scric as highly clficient procedures to preparc Claisen type $\beta$-hydroxyester products either by in situ or subsequent acid-catalyzed methanolysis of the initially lormed 2.2-dioxyoxetanes. Examples which provide support for this conclusion are given in Scheme 4 .

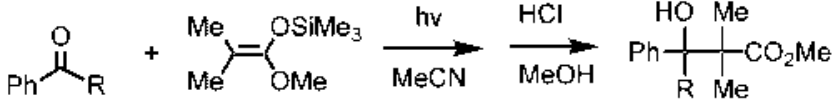

$$
\begin{aligned}
& 1(\mathrm{R}=\mathrm{H}) \\
& 2(\mathrm{R}=\mathrm{Me}) \\
& 9 \\
& 13(\mathrm{R}=\mathrm{H}) \\
& 14(\mathrm{R}=\mathrm{Me})
\end{aligned}
$$

Scheme 4

\section{Experimental Section}

General Procedure. The chemical shifts of resonances in the ${ }^{1} \mathrm{H}-\mathrm{NMR}(200)$ and $\left.300 \mathrm{MH} / 2\right)$ and ${ }^{13} \mathrm{C}$-NMR $(50$ and 75 $\mathrm{MH} /$ ) spectra were recorded on $\mathrm{CDCl}_{3}$ solutions are reported in parts per million relative to $\mathrm{Me}_{+} \mathrm{Si}$ as an internal standard. For compounds containing $\mathrm{Me}_{3} \mathrm{Si}$ groups. $\mathrm{CHCl}_{3}$ was used as an internal standard. ${ }^{1.9} \mathrm{C}-\mathrm{NMR}$ resonances were assigned by use of the DEPT technique to determine the numbers of attached hydrogens. IR spectral bands are reported in $\mathrm{cm}^{-1}$. Preparative photochemical reactions were conducted with an apparatus consisting of a $450 \mathrm{~W}$ Hanovia medium pressure mercury vapor lamp surrounded by a Pyrex glass filter in a water-cooled quartz inunersion well surrounded by the solution being irradiated. The photoly sis solutions were purged with nitrogen before and during irradiations. The photolysates were concentrated under reduced pressure giving residues which were subjected to preparative TLC on $20 \times 20 \mathrm{~cm}$ silica gel coated plates. Low and high resolution (HRMS) mass spectra were obtained by use of electron impact ionization unless otherwise noted. All starting materials used in the photoreactions derived from commercial sources. All new compounds described are isolated as oils in $>90 \%$ purity (by NMR analysis) unless noted otherwise.

Irradiation of Benzaldehyde (1) and 1-Trimethylsilyloxy1-methoxy-2,2-dimethylethylene (9). Acetonitrile. A 
solution of benzaldehy de (1) (382 $\mathrm{mg} .3 .6 \mathrm{mmol}$ ) and ketene acctal 9 (1.26 g. $7.2 \mathrm{mmol}$ ) in $100 \mathrm{~mL}$ of acetonitrile was irradiated for $3 \mathrm{~h}(c a .98 \%$ conversion of 1 ). Work-up and chromatographic ( $1: 8$. cthy 1 acctate $: n$-hexane. $v / s)$ scparation (sec General) gave $556 \mathrm{mg}$ (76\%) of 13 and $45 \mathrm{mg}$ (5\%) of 31. A solution of oxctanc 31 (100 $\mathrm{mg} .0 .36 \mathrm{mmol})$ and trace of $5 \% \mathrm{HCl}$ in $50 \mathrm{~mL}$ of methanol was stirred for $4 \mathrm{~h}$ at $25^{\circ} \mathrm{C}$. Work-up and chromatographic $(1: 8$. cthyl acetate $n$-hexanc. $v / v$ ) separation (sec General) gave $76 \mathrm{mg}(76 \%)$ of 13.

13: mp 65-67 $7^{\circ} \mathrm{C}$. ${ }^{1} \mathrm{H}$ NMR 1.10 (s. $\left.3 \mathrm{H} . \mathrm{C}\left(\mathrm{CH}_{3}\right)_{2}\right) .1 .14$ (s. $\left.3 \mathrm{H} . \mathrm{C}\left(\mathrm{CH}_{3}\right)\right)_{2} .37$ (s. IH. OH). $3.7 \mathrm{l}\left(\mathrm{s} .3 \mathrm{H}_{0} \mathrm{OCH}_{3}\right) .4 .89(\mathrm{~d}$. $1 \mathrm{H} . J=3.7 \mathrm{H} / \mathrm{C}(\mathrm{OH}) \underline{\mathrm{H}}) .7 .27-7.37(\mathrm{~m} .5 \mathrm{H}$ aromatic $):{ }^{13} \mathrm{C}$ NMR $19.0\left(\mathrm{C}\left(\mathrm{CH}_{3}\right)_{2}\right) .22 .9\left(\mathrm{C}\left(\mathrm{CH}_{3}\right)_{2}\right) .47 .7\left(\mathrm{C}\left(\mathrm{CH}_{3}\right)_{2}\right) .52 .0$ $\left(\mathrm{OCH}_{3}\right) .78 .6(\mathrm{C}(\mathrm{OH}) \mathrm{H}) .127 .6$ and 127.7. $(\mathrm{CH}$. aromalic). 140.0 (C. aromatic). 178. $1(\mathrm{C}=\mathrm{O})$ : Ir $(\mathrm{KBr}) .3350-3520$ (br. OH stretching). $1700 \mathrm{~cm}^{-1}(\mathrm{C}=\mathrm{O}$ streching): CIMS. $\mathrm{m} / \%$ (rel. intensity) $209(\mathrm{M}+\mathrm{l} .1) .191(\mathrm{M}-\mathrm{OH} .10) .149(2) .132(3)$. $107(28) .102(100) .77(22)$ : HRMS. $\mathrm{m} / \mathrm{t} .209 .1185\left(\mathrm{C}_{12} \mathrm{H}_{1} 7 \mathrm{O}_{3}\right.$ requires 209.1178$)$.

31: ${ }^{1} \mathrm{H}$ NMR -0.05 (s. 9H. OSiMc 3 ). 1.41 (s. $3 \mathrm{H} . \mathrm{C}\left(\mathrm{CH}_{3}\right)$ ). 1.52 (s. 3H. $\mathrm{C}\left(\mathrm{CH}_{3}\right)$ ). 3.39 (s. $\left.3 \mathrm{H} . \mathrm{OCH}_{3}\right) .5 .57$ (s. $1 \mathrm{H}$. $\mathrm{C}(\mathrm{Ph}) \underline{\mathrm{H}}) .7 .27-7.3 \%$ (m. 5H. aromatic): ${ }^{13} \mathrm{C}$ NMR $\mathrm{l} . \mathrm{l}\left(\mathrm{OSiMc}_{3}\right)$. $\left.\left.23.8\left(\mathrm{C}\left(\mathrm{CH}_{3}\right)_{2}\right) .24 .7\left(\mathrm{C}_{(\mathrm{C}} \mathrm{C}_{3}\right)_{2}\right) .51 .2\left(\mathrm{OCH}_{3}\right) .87 .4\left(\mathrm{C}_{(\mathrm{CH}}\right)_{2}\right)$. $91.3(\mathrm{C}(\mathrm{Pl}) \mathrm{H}) .102 .9\left(\mathrm{C}\left(\mathrm{OCH}_{3}\right) \mathrm{OSiMc} 3\right) .127 .3 .127 .6$ and 127.7 (CH. aromatic). 138.0 (C. aromatic): Ir (ncat). 1250 $\mathrm{cm}^{-1}$ (C-O stretching): EIMS. m/r. (rel. intensily) 280 (M'. 0.1). 222 (13). 174 (100). 159 (22). $118(94) .105$ (98): HRMS. $\mathrm{m} /, 280.1491\left(\mathrm{C}_{15} \mathrm{H}_{2+} \mathrm{O}_{3} \mathrm{Si}\right.$ rcquires 280.1495$)$.

Benzene. A solution of benzaldelyde (1) (382 mg. 3.6 mmol) and ketene acctal 9 (1.26 g. $7.2 \mathrm{mmol})$ in $100 \mathrm{~mL}$ of benzene was irradiated for 11 h ( $c a .7 \% \%$ conversion of 1 ). Work-up and chromatographic ( $1: 8$. chly l acctate $: n$-hexanc. v/r) separation (sce Gencral) gave $424 \mathrm{mg}(72 \%)$ of 13.55 $\mathrm{mg}(8 \%)$ of $31.28 \mathrm{mg}(1 \%)$ of 27 . and $11 \mathrm{l} \mathrm{mg}(14 \%)$ of $\mathbf{4 2}$.

27: ${ }^{1} \mathrm{H}$ NMR 0.24 (s. 9H. OSiMc $\mathrm{S}_{3}$ ). 1.48 (s. $3 \mathrm{H} . \mathrm{C}\left(\mathrm{CH}_{3}\right)$ ). 1.50 (s. $\left.3 \mathrm{H} . \mathrm{C}\left(\mathrm{CH}_{3}\right)_{2}\right) .2 .88$ (s. $3 \mathrm{H}, \mathrm{OCH}_{3}$ ). 5.49 (s. $\mathrm{lH}$. $\mathrm{C}(\mathrm{Ph}) \underline{\mathrm{H}}) .7 .32-7.49$ (s. $5 \mathrm{H}$. aromatic).

42: ' $\mathrm{H}$ NMR -0.04 (s, 9H. OSiMe 3 ), 0.99 (s. 3H, C( $\left.\left.\mathrm{CH}_{3}\right)_{2}\right)$. 1.13 (s. $\left.3 \mathrm{H} . \mathrm{C}\left(\mathrm{CH}_{3}\right)_{2}\right) .3 .68$ (s. $\left.3 \mathrm{H} . \mathrm{OCH}_{3}\right), 4.97$ (s. $1 \mathrm{H}$. $\mathrm{C}(\mathrm{Ph}) \underline{\mathrm{H}}) .7 .27$ (s. $5 \mathrm{H}$. aromatic): ${ }^{13} \mathrm{C}$ NMR $-0.1\left(\mathrm{OSiMe}_{3}\right)$. $\left.19.1\left(\mathrm{C}\left(\mathrm{CH}_{3}\right)_{2}\right) .21 .7\left(\mathrm{C}\left(\mathrm{CH}_{3}\right)\right), 49.0\left(\mathrm{C}\left(\mathrm{CH}_{3}\right)\right)_{2}\right) .51 .6\left(\mathrm{OCH}_{3}\right)$, $79.1(\mathrm{C}(\mathrm{Ph}) \mathrm{H}), 127.4,127.8$ and $127.8(\mathrm{CH}$ aromatic). 140.8 (C. aromatic), $177.3(\mathrm{C}=\mathrm{O})$; $\operatorname{Ir}($ neat $) 1810(\mathrm{C}=\mathrm{O}$ stretching $)$, $1250 \mathrm{~cm}^{-1}$ (C-O stretching): EIMS. $\mathrm{m} / \mathrm{z}$ (rel. intensity) 279 $\left(\mathrm{M}^{+}, 19\right), 265$ (4), 235 (1). 213 (2), 205 (3), 179 (100), 174 (23). 107 (46): HRMS、279.1383 $\left(\mathrm{C}_{1:} \mathrm{H}_{33} \mathrm{O}_{3} \mathrm{Si}\right.$ requires 279.1417).

Irradiation of Acetophenone (2) and 1-Trimethylsilyloxy-1-methoxy-2,2-dimethylethylene (9). Acetonitrile. A solution of acetophenone (2) $(430 \mathrm{mg} .3 .6 \mathrm{mmol})$ and ketene acetal 9 (1.26 g. $7.2 \mathrm{mmol}$ ) in $100 \mathrm{~mL}$ of acetonitrile was irradiated for 32 h ( $c a .100 \%$ conversion of 2$)$. Work-up and cluromatographic ( $1: 8$. ethyl acetate $: n$-hexane. $v / v)$ separation (see General) gave the diastereomeric oxetanes 32a $(55 \mathrm{mg} .5 \%)$ and $32 \mathrm{~b}(65 \mathrm{mg} .6 \%) .666 \mathrm{mg}(83 \%)$ of 28 . and $22 \mathrm{mg}(5 \%)$ of diol 47 . A solution of oxetane $32(100 \mathrm{mg}$.
$0.34 \mathrm{mmol}$ ) and trace of $5 \% \mathrm{HCl}$ in $50 \mathrm{~mL}$ of methanol was stirred for 4 ht at $25^{\circ} \mathrm{C}$. Work-up and chromatographic $(1: 8$. cthyl acctate: $n$-hexane. v/v) scparation (sce General) gave $83 \mathrm{mg}(83 \%)$ of 14 .

28: ${ }^{1} \mathrm{H}$ NMR 1.15 (s. 3H, C(CH $\left.)_{3}\right), 1.16\left(\mathrm{~s} .3 \mathrm{H}, \mathrm{C}\left(\mathrm{CH}_{3}\right)_{2}\right)$. 1.61 (s. $3 \mathrm{H} . \mathrm{C}\left(\mathrm{CH}_{3}\right) \mathrm{OH}$ ). 3.67 (s. $3 \mathrm{H} . \mathrm{OCH}_{3}$ ). 4.39 (s. $1 \mathrm{H}$. $\mathrm{OH})$. 7.23-7.47 (m. 5H. aromatic): ${ }^{13} \mathrm{C}$ NMR (acctonc-d $) 22.2$ $\left(\mathrm{C}\left(\mathrm{CH}_{3}\right)_{2}\right) .22 .4\left(\mathrm{C}\left(\mathrm{CH}_{3}\right)_{2}\right) .26 .1\left(\mathrm{C}(\mathrm{OH}) \mathrm{CH}_{3}\right) .51 .6\left(\mathrm{C}\left(\mathrm{CH}_{3}\right)_{2}\right)$. $52.3\left(\mathrm{OCH}_{3}\right), 77.5\left(\mathrm{C}(\mathrm{OH}) \mathrm{CH}_{3}\right), 127.6,128.0$ and 128.1 $(\mathrm{CH}$. aromatic). $146.2(\mathrm{C}$. aromatic). $178.3(\mathrm{C}=\mathrm{O})$ : $\operatorname{lr}($ neat $)$. $3300-3600$ (br. OH stretching). $1700(\mathrm{C}=\mathrm{O}$ stretching). 1280 (C-O stretching): EIMS. m// (rcl.intensity) $222\left(\mathrm{M}^{\prime}\right.$, I). 205 (M'-OH. 15), $189(4), 145(3) .121$ (100). $105(16) .102(66)$. 77 (8): HRMS. $\mathrm{m} / \mathrm{t} .222 .1260\left(\mathrm{C}_{13} \mathrm{H}_{18} \mathrm{O}_{3}\right.$ requires 222.1256$)$.

32a: ${ }^{1} \mathrm{H}$ NMR -0.07 (s. 9H. OSiMc 3 ), 1.28 (s. 3H. C(CH $\left.{ }_{3}\right)_{2}$ ). 1.49 (s. $\left.3 \mathrm{H} . \mathrm{C}\left(\mathrm{CH}_{3}\right)_{2}\right), 1.67$ (s. $3 \mathrm{H} . \mathrm{C}\left(\mathrm{CH}_{3}\right) \mathrm{Ph}$ ). 3.4 (s. $3 \mathrm{H}$. $\left.\mathrm{OCH}_{3}\right) .7 .25-7.40$ (m. 5H. aromatic): ${ }^{13} \mathrm{C}$ NMR I.1 (OSiMc $\left.)_{3}\right)$. $24.9\left(\mathrm{C}\left(\mathrm{CH}_{3}\right)_{2}\right) .25 .9\left(\mathrm{C}\left(\mathrm{CH}_{3}\right)\right) .26 .7\left(\mathrm{C}\left(\mathrm{CH}_{3}\right) \mathrm{Ph}\right) .51 .6\left(\mathrm{OCH}_{3}\right)$. $89.1\left(\underline{\mathrm{C}}\left(\mathrm{CH}_{3}\right)_{2}\right) .92 .3\left(\mathrm{C}\left(\mathrm{CH}_{3}\right) \mathrm{Pl}\right) .103 .0\left(\mathrm{C}_{(}\left(\mathrm{OCH}_{3}\right) \mathrm{OSiMc}_{3}\right)$. 125.7. 126.3 and 127.4 (CH. aromatic). $1+4.4$ (C. aromatic): Ir (ncat) 1250 (C-O stretching): CIMS, m/ $/$ (rcl. intensity) $295\left(\mathrm{M}^{\prime}+1,2\right) .236(\mathrm{l} 0) .193(8) .132(+3) .105$ (43): HRMS. $\mathrm{m} / .295 .1715\left(\mathrm{C}_{16} \mathrm{H}_{27} \mathrm{O}_{3} \mathrm{Si}\right.$ requires 295.1729$)$.

32b: 'H NMR 0.28 (s. 9H. OSiMc 3 ). 1.42 (s. 3H. C( $\left(\mathrm{CH}_{3}\right)$ ). 1.48 (s. $\left.3 \mathrm{H} . \mathrm{C}\left(\mathrm{CH}_{3}\right)_{2}\right) .1 .67$ (s. $\left.3 \mathrm{H} . \mathrm{C}\left(\mathrm{CH}_{3}\right) \mathrm{Ph}\right) .2 .89$ (s. $3 \mathrm{H}$. $\left.\mathrm{OCH}_{3}\right) .7 .2 \mathrm{l}-7.5 \mathrm{l}\left(\mathrm{m} .5 \mathrm{H}\right.$. aromatic): ${ }^{13} \mathrm{C}$ NMR I.6 $\left(\mathrm{OSiMc}_{3}\right)$. $\left.\left.24.3\left(\mathrm{C}\left(\mathrm{CH}_{3}\right)_{2}\right) \cdot 26.5\left(\mathrm{C}\left(\mathrm{CH}_{3}\right)\right)_{2}\right) .26 .6\left(\mathrm{C}_{(\mathrm{CH}}\right) \mathrm{Ph}\right) .51 .2\left(\mathrm{OCH}_{3}\right)$. $89.6\left(\underline{\mathrm{C}}\left(\mathrm{CH}_{3}\right)_{2}\right) .91 .2\left(\underline{\mathrm{C}}\left(\mathrm{CH}_{3}\right) \mathrm{Ph}\right) .103 .2\left(\underline{\mathrm{C}}\left(\mathrm{OCH}_{3}\right) \mathrm{OSiMc}_{3}\right)$. 126.2. 126.7 and $127.4(\mathrm{CH}$. aromatic). $1+3.8$ (C.aromatic): Ir(ncal) 1260 (C-O stretching): CIMS. $\mathrm{m} / \%$ (rcl. intensity) $295(\mathrm{M}+\mathrm{l} . \mathrm{l}) .279$ (2). 247 (2). 236 (1l). 132 (52). 105 (I0): HRMS. m/. 295.1704 $\left(\mathrm{C}_{16} \mathrm{H}_{27} \mathrm{O}_{3} \mathrm{Si}\right.$ requires 295.1729$)$.

Benzenc. A solution of acctophenone (2) (430) $\mathrm{mg} .3 .6$ mmol) and ketenc acetal $9(1.26 \mathrm{~g} .7 .2 \mathrm{mmol})$ in $100 \mathrm{~mL}$ of ben/ene was irradiated for $33 \mathrm{~h}$. ( $\mathrm{ca} .77 \%$ conversion of 2 ) Work-up and chromatographic ( $1: 20$. cthyl acctate : $n$-hexanc. v/v) scparation (sce Gencral) gave $392 \mathrm{mg}(64 \%)$ of 28.90 $\mathrm{mg}(11 \%)$ of $32 \mathrm{a} .57 \mathrm{mg}(7 \%)$ of $32 \mathrm{~b}$ and $8 \mathrm{mg}(3 \%)$ of diol 47.

Irradiation of Benzophenone (3) and 1-Trimethylsilyloxy-1-methoxy-2,2-dimethylethylene (9). Acetonitrile. A solution of benzophenone (2) (656 mig. $3.6 \mathrm{mmol}$ ) and ketene acetal $9(1.26 \mathrm{~g} .7 .2 \mathrm{~mm}$ ol) in $100 \mathrm{~mL}$ of acetonitrile was irradiated for $6 \mathrm{~h}$ (ca. $100 \%$ conversion of 3 ). Work-up and chromatographic $(1: 8$. etlyl acetate $: n$-hexane, $v / v)$ separation (see General) gave $130 \mathrm{mg}(13 \%)$ of $15.840 \mathrm{mg}$ $(65 \%)$ of 29 and $150 \mathrm{mg}(23 \%)$ of benzopinacole 48 .

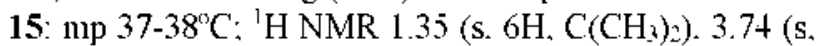
$\left.3 \mathrm{H} . \mathrm{OCH}_{3}\right) .5 .08$ (s. 1H. OH). 7.23-7.37 (nt. 10H. aromatic): ${ }^{1.3} \mathrm{C}$ NMR $24.0\left(\mathrm{C}\left(\mathrm{CH}_{3}\right)_{2}\right) .49 .0\left(\mathrm{C}\left(\mathrm{CH}_{3}\right)_{2}\right), 52.5\left(\mathrm{OCH}_{3}\right)$. $82.2(\mathrm{COH}), 126.9 .127 .2$ and $128.6(\mathrm{CH}$ arontatic) 145.2 (C. aromatic). $180.3(\mathrm{C}=\mathrm{O})$ : Ir $(\mathrm{KBr}) 3350-3550$ (br. $\mathrm{OH}$ stretching). $1700\left(\mathrm{C}=\mathrm{O}\right.$ stretcluing). $1270 \mathrm{cml}^{-1}$ (C-O stretcling): EIMS, $\mathrm{m} / \mathrm{z}$ (rel. intensity). $284\left(\mathrm{M}^{+}, 0.1\right), 267(38) .183$ (100). $105(98) .77$ (33): HRMS. $1 \mathrm{~m} / \mathrm{z} 284.1419\left(\mathrm{C}_{18} \mathrm{H}_{20} \mathrm{O}_{3}\right.$ requires $28+.1+12$ ).

29: 'H NMR 0.30 (s. 9H. OSiMe 3 ). 1.19 (s. $\left.3 \mathrm{H} . \mathrm{C}\left(\mathrm{CH}_{3}\right)_{2}\right)$ ). 
$1.20\left(\mathrm{~s}, 3 \mathrm{H}, \mathrm{C}\left(\mathrm{CH}_{3}\right)\right), 3.46\left(\mathrm{~s} .3 \mathrm{H}, \mathrm{OCH}_{3}\right), 7.22-7.63$ (m,

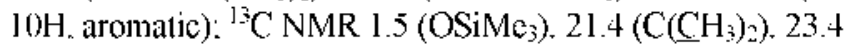
$\left(\mathrm{C}\left(\mathrm{CH}_{3}\right)_{2}\right) .48 .6\left(\underline{\mathrm{C}}\left(\mathrm{CH}_{3}\right)_{2}\right) .53 .6\left(\mathrm{OCH}_{3}\right) .86 .1\left(\underline{\mathrm{C}}(\mathrm{Ph})_{2}\right) .1150$ $\left(\underline{\mathrm{C}}\left(\mathrm{OCH}_{3}\right) \mathrm{OSiMc}_{3}\right), 125.5,125.7,126.3,126.3,127.5$ and $127.6\left(\mathrm{CH}_{\text {. aromatic) }} \mathrm{l} 44\right.$, and $144.3(\mathrm{C}$. aromatic): Ir(ncat) $1260 \mathrm{~cm}^{-1}$ (C-O stretching): CIMS. m/ (rel intensity) 357 $\left(\mathrm{M}^{\prime}+\mathrm{l}, 0.1\right) .34 \mathrm{l}(4) .325(6) .281$ (23). $255(58) .208(71)$. 183 (57). 105 (100). 77 (32); HRMS, m/\% 357.1882 $\left(\mathrm{C}_{23} \mathrm{H}_{2}, \mathrm{O}_{3} \mathrm{Si}\right.$ requires 357.1886$)$.

Benzene. A solution of benzophenone (3) $(656 \mathrm{mg} .3 .6$ mmol) and ketene accial $9(1.26 \mathrm{~g} .7 .2 \mathrm{mmol})$ in $100 \mathrm{~mL}$ of benzene was irradiated for $4 \mathrm{~h}(\mathrm{ca} .95 \%$ conversion of 3 ). Work-up and chromatographic ( $1: 8$, ethyl acclate : $n$-hexanc. v/v) separation (see Gencral) gave $104 \mathrm{mg}$ ( $11 \%$ ) of 15.325 $\mathrm{mg}(27 \%)$ of $29.33 \mathrm{mg}(3 \%)$ of 33 and $248 \mathrm{mg}(40 \%)$ of ben/opinacole $\mathbf{4 8}$.

33: mp 100-102 "C: ${ }^{\circ} \mathrm{H}$ NMR 0.07 (s.9H, OSiMc 3 ). 1.29 (s. $\left.3 \mathrm{H}, \mathrm{C}\left(\mathrm{CH}_{3}\right)_{2}\right) .1 .53$ (s. $3 \mathrm{H}_{2} \mathrm{C}\left(\mathrm{CH}_{3}\right)$ ) 2.86 (s. $\left.3 \mathrm{H}, \mathrm{OCH}_{3}\right)$. 7.26-7.60 (m. 10H, aromatic): ${ }^{13} \mathrm{C}$ NMR l.2 (OSiMc 3$) .23 .3$ $\left.\left(\mathrm{C}^{\left(\mathrm{CH}_{3}\right.}\right)_{2}\right)_{2} 25.8\left(\mathrm{C}\left(\mathrm{CH}_{3}\right)_{2}\right) .51 .0\left(\mathrm{OCH}_{3}\right) .89 .8\left(\mathrm{C}\left(\mathrm{CH}_{3}\right)_{2}\right) .93 .8$ $\left(\underline{\mathrm{C}}(\mathrm{Ph})_{2}\right) .104 .5\left(\mathrm{C}\left(\mathrm{OCH}_{3}\right) \mathrm{OSSMM}_{3}\right) .126,6,126.9 .127,0.127 .3$. 128.0 and $128 . \mathrm{l}(\mathrm{CH}$. aromatic). 141.8 and $\mathrm{l}+2.2(\mathrm{C}$. aromatic): $\operatorname{lr}$ (neat) $1250 \mathrm{~cm}^{-1}$ (C-O. strclching): EIMS. $\mathrm{m} / \mathrm{t}$ (rel. intensity) 356 (M .0.04). 288 (4). 256 (2). 194 (17). $182(9), 176(5), 174(100), 166(14)$ : HRMS. $m / / 356.1820$ $\left(\mathrm{C}_{21} \mathrm{H}_{28} \mathrm{O}_{3} \mathrm{Si}\right.$ requires 356,1808$)$.

Irradiation of 4 -Methoxyacetophenone (4) and 1-Trimethỵlsilyloxy-1-methoxy-2,2-dimethỵlethỵlene (9). Acetonitrile. A solution of 4'-methoxyacetophenone (4) (540 mg. $3.6 \mathrm{mmol})$ and ketene acclal $9(\mathrm{l} .26 \mathrm{~g} .7 .2 \mathrm{mmol})$ in $100 \mathrm{~mL}$ of acctonitrile was irradiated for 43 h (ca. $74 \%$ conversion of 4). Work-up and chromatographic ( $\mathrm{CHCl}_{3}$ ) scparation (sce General) gave $391 \mathrm{mg}(62 \%)$ of 16 .

16: mp 82-84 "C: 'H NMR 1.14 (s. 6H. C(C (CH3) $) .1 .57$ (s. $3 \mathrm{H} . \mathrm{C}(\mathrm{OH}) \mathrm{CH}_{3}$ ). 3.68 (s. 3H. COC CH$)_{3} .3 .79$ (s. $3 \mathrm{H} . \mathrm{PhOC}_{3}$ ). 6.84 (d. $2 \mathrm{H} . J=9.0 \mathrm{H} \%$ aromatic). 7.36 (d. $2 \mathrm{H} . J=9.0 \mathrm{H} \%$ aromatic): ${ }^{13} \mathrm{C}$ NMR $21.6\left(\mathrm{C}\left(\mathrm{CH}_{3}\right)_{2}\right) \cdot 21.7\left(\mathrm{C}\left(\underline{\mathrm{CH}}_{3}\right)_{2}\right) .25 . \mathrm{l}$ $(\mathrm{C}(\mathrm{OH}) \underline{\mathrm{CH}} 3), 50.4\left(\underline{\mathrm{C}}\left(\mathrm{CH}_{3}\right)_{2}\right), 52.1\left(\mathrm{CO}_{2} \mathrm{CH}_{3}\right) .55 .1\left(\mathrm{PlOCH}_{3}\right)$, $76.8\left(\mathrm{C}(\mathrm{OH}) \mathrm{CH}_{3}\right) .112 .5$ and $128.3(\mathrm{CH}$ aromatic) 135.6 and $158 .+(\mathrm{C}$. aromatic), $179.0(\mathrm{C}=\mathrm{O})$; Ir $(\mathrm{KBr}) 3280-3500$ (br, $\mathrm{OH}$ stretching), $1700 \mathrm{~cm}^{-1}(\mathrm{C}=\mathrm{O}$ stretching); EIMS, $\mathrm{m} / \mathrm{z}$ (rel. intensity). $236\left(\mathrm{M}^{-}, 2\right), 235$ (13). 219 (3). 175 (2), 151 (100). 135 (24). 92 (5): HRMS. m/z $236.1411\left(\mathrm{C}_{14} \mathrm{H}_{2} \mathrm{O}_{3}\right.$ requires 236.1413 ).

Irradiation of 4-Cyanoacetophenone (5) and 1-Trimethylsilyloxy-1-methoxy-2,2-dimethylethylene (9). Acetonitrile. A solution of 4-cyanoacetophenone (5) $(520 \mathrm{mg} .3 .6 \mathrm{mmol})$ and ketene acetal 9 (1.26 g, $7.2 \mathrm{mmol})$ in $100 \mathrm{~mL}$ of acetonitrile was irradiated for $28 \mathrm{~h}(\mathrm{ca} .88 \%$ conversion of 5). Work-up and chromatographic ( $1: 5$, ethyl acetate : $n$ hexane, $\%$ ) separation (see General) gave $246 \mathrm{mg}(32 \%)$ of 17. $104 \mathrm{mg}(10 \%)$ of $\mathbf{4 3}$ and $158 \mathrm{mg}(34 \%)$ of 49 .

17: ${ }^{1} \mathrm{H}$ NMR 1.10 (s. 3H. C $\left.\left(\mathrm{CH}_{3}\right)_{2}\right)$. 1.13 (s. $\left.3 \mathrm{H} . \mathrm{C}\left(\mathrm{CH}_{3}\right)_{2}\right)$. 1.59 (s, $\left.3 \mathrm{H}, \mathrm{C}(\mathrm{OH}) \underline{H}_{3}\right), 3.68$ (s. $\left.3 \mathrm{H}, \mathrm{OCH}_{3}\right), 4.52$ (s. $1 \mathrm{H}$. $\mathrm{OH}), 7.57$ (s, $4 \mathrm{H}$, aromatic): ${ }^{1.3} \mathrm{C}$ NMR (acetone- $\left.\mathrm{d}_{6}\right) 22.1$ $\left(\mathrm{C}\left(\mathrm{CH}_{3}\right)_{2}\right) .22 .4\left(\mathrm{C}\left(\underline{\mathrm{CH}}_{3}\right)\right) .25 .9\left(\mathrm{C}(\mathrm{OH}) \underline{C H}_{3}\right) .51 .8\left(\mathrm{C}\left(\mathrm{CH}_{3}\right)_{2}\right)$. $52.5\left(\mathrm{OCH}_{3}\right) .77 .7\left(\mathrm{C}(\mathrm{OH}) \mathrm{CH}_{3}\right) .111 .7(\mathrm{CN}) .119 .8$ and 152.2
(C. aromatic). 129.2 and $132.1(\mathrm{CH}$ aromatic). $177.7(\mathrm{C}=\mathrm{O})$; Ir (neat). 3250-3600 (br. OH stretching). 2230 (CN stretching). $1720 \mathrm{~cm}^{-1}$ (C=O stretching): C.IMS. $\mathrm{m} / \%$ (rcl, intensity) $248\left(\mathrm{M}^{-}+1,26\right), 230(\mathrm{M}-\mathrm{OH}, 41), 146(43), 130(26), \mathrm{l}(12$ (100): HRMS. m/\%. $248.1290\left(\mathrm{C}_{14} \mathrm{H}_{18} \mathrm{NO}_{3}\right.$ requires 248.1287$)$.

43. ${ }^{l} \mathrm{H}$ NMR $0.06\left(\mathrm{~s} .9 \mathrm{H} . \mathrm{OSiMc}_{3}\right), 1.06\left(\mathrm{~s} .3 \mathrm{H}, \mathrm{C}\left(\mathrm{CH}_{3}\right)_{2}\right)$. $1.13\left(\mathrm{~s}, 3 \mathrm{H}, \mathrm{C}\left(\mathrm{CH}_{3}\right)_{2}\right), 1.78$ (s. $3 \mathrm{H},\left(\mathrm{C}\left(\mathrm{OSiMc}_{3}\right) \mathrm{CH}_{3}\right) .3 .54$ (s. $\left.3 \mathrm{H}_{2} \mathrm{OCH}_{3}\right) .7 .43$ (d. $2 \mathrm{H} . J=8.4 \mathrm{H} /$. aromatic). 7.59 (d. $2 \mathrm{H}, J=8.4 \mathrm{H} \%$ aromatic): ${ }^{13} \mathrm{C}$ NMR $2.5\left(\mathrm{OSiMc}_{3}\right) .21 .6$ $\left(\mathrm{C}\left(\mathrm{CH}_{3}\right)_{2}\right), 22.1 \quad\left(\mathrm{C}\left(\mathrm{CH}_{3}\right)_{2}\right), 25.0\left(\mathrm{C}\left(\mathrm{OSiMc}_{3}\right) \mathrm{CH}_{3}\right), 51.9$ $\left(\underline{\mathrm{C}}\left(\mathrm{CH}_{3}\right)_{2}\right) .52 .7\left(\mathrm{OCH}_{3}\right) .80 .6\left(\mathrm{C}\left(\mathrm{OSiMc}_{3}\right) \mathrm{CH}_{3}\right) .111 .2(\mathrm{CN})$. 119.4 and 151.5 (C. aromatic), $128 . \mathrm{I}$ and $13 \mathrm{l} .4(\mathrm{CH}$. aromatic). 176,4 (C=O): Ir(ncat) 2230 ( $\mathrm{CN}$ strctching). 1730 $\mathrm{cm}^{-1}(\mathrm{C}=\mathrm{O}$ stretching): CIMS, $\mathrm{m} / \%$ ( $\mathrm{rcl}$, intensity). 320 $(\mathrm{M}+\mathrm{l}, 3), 304(8), 29 \mathrm{l}(9), 218(\mathrm{l} 00), 89$ (2). 73 (9): HRMS. m/. $320.1682\left(\mathrm{C}_{17} \mathrm{H}_{26} \mathrm{NO}_{3} \mathrm{Si}\right.$ requires 320.1682$)$.

49: mp 22 l-223 ${ }^{\circ} \mathrm{C}$ : ' $\mathrm{H}$ NMR (DMSO-d $\mathrm{d}_{i}$ ) 1.23 (s. $3 \mathrm{H}$. $\mathrm{CH}_{3}$ ). 1.62 (s. $\left.3 \mathrm{H} . \mathrm{CH}_{3}\right) .5 .37$ (s. lH. OH). 5.55 (s. IH. OH). 7.24 (d. $2 \mathrm{H} . J=8.0 \mathrm{H} /$. aromatic). 7.51 (d. $2 \mathrm{H} . J=8.0 \mathrm{H} \%$ aromatic). 7.67 (d. $2 \mathrm{H} . J=8.0 \mathrm{H} /$. aromatic). 7.74 (d. $2 \mathrm{H} . J$ $=8.0 \mathrm{H} \%$ aromatic): ${ }^{13} \mathrm{C}$ NMR $($ DMSO-d $) 24.5$ and 24.8 $\left(\mathrm{C}(\mathrm{OH}) \mathrm{CH}_{3}\right), 76.9$ and $77.2\left(\underline{\mathrm{C}}(\mathrm{OH}) \mathrm{CH}_{3}\right), \mathrm{I} 08.7$ and 109.1 (CN). 128. I. 128.8. 130.3 and 130.7 (CH. aromatic). 152.2 and 152,6 (C. aromatic): Ir (KBr) $3200-3600$ (br. $\mathrm{OH}$ strelching). $2230 \mathrm{~cm}^{-1}$ (CN strctching): CIMS. $\mathrm{m} / \mathrm{x}$ ( $\mathrm{rcl}$. intensity) $293(\mathrm{M}+1.3) .258(6) .147(\mathrm{l} 00) .130(50)$ : HRMS. $\mathrm{m} / 1.293 .1281$ ( $\mathrm{C}_{18} \mathrm{H}_{17} \mathrm{~N}_{2} \mathrm{O}_{2}$ requires 293.1290 ).

Benzene. A solution of 4-cyanoacclophenone (5) (520 mg. $3.6 \mathrm{mmol})$ and ketene acctal $9(\mathrm{l} .26 \mathrm{~g} .7 .2 \mathrm{mmol})$ in $\mathrm{l}(00$ $\mathrm{mL}$ of benzene was irradiated for $10 \mathrm{~h}$ (ca. $86 \%$ conversion of 5). Work-up and chromatographic ( $1: 15$. chly l acctatc : $n$-hexanc. $\mathrm{V} / \mathrm{r}$ ) separation (sce Gencral) gave $652 \mathrm{mg}(86 \%)$ of 17 . trace of 43 and $21 \mathrm{mg}(5 \%)$ of 49 .

$35 \% \mathrm{H}_{2} \mathrm{O}$-Acetonitrile. A solution of 4-cyanoacctophenone (5) (520 $\mathrm{mg} .3 .6 \mathrm{mmol}$ ) and ketenc acctal 9 (1.26 g. 7.2 mmol)) in a solution of $65 \mathrm{~mL}$ of acctonitrilc and $35 \mathrm{~mL}$ of $\mathrm{H}_{2} \mathrm{O}$ was irradiated for $37 \mathrm{~h}$ ( $c a$. $62 \%$ conversion of 5 ). Work-up and chromatographic (1:8, ethyl acetate: $n$ hexame. $v / v$ ) separation (see General) gave $60 \mathrm{mg}(11 \%)$ of $17.20 \mathrm{mg}(3 \%)$ of 43 and $235 \mathrm{mg}(72 \%)$ of 49.

Irradiation of Butyrophenone (6) and 1-Trimethylsilyloxy-1-methoxy-2,2-dimethylethylene (9). Acetonitrile. A solution of buty rophenone (6) $(500 \mathrm{mg} .3 .4 \mathrm{~mm}$. ketene acetal $9(1.18 \mathrm{~g} .6 .8 \mathrm{mmol})$ in $100 \mathrm{~mL}$ of acetonitrile was irradiated for $9 \mathrm{~h} \mathrm{(ca.} 100 \%$ conversion of 6 ). Work-up and chromatographic $(1: 8$. etlyl acetate: $n$-hexane, $v / v)$ separation (see General) gave the diastreomeric oxetanes $28 \mathrm{a}(30 \mathrm{mg}$. $3 \%$ ) and $28 \mathrm{~h}(30 \mathrm{mg}$. 3\%). 41 a $(33 \mathrm{mlg} .3 \%)$ and $41 \mathrm{~b}(45 \mathrm{mg} .4 \%) .184 \mathrm{mg}(45 \%)$ of $2.20 \mathrm{mg}(4 \%)$ of 50 . $247 \mathrm{mg}(29 \%)$ of 18 . and $55 \mathrm{mlg}(5 \%)$ of 30 .

18: ${ }^{1} \mathrm{H}$ NMR 0.85 (t. $3 \mathrm{H} . J=6.6 \mathrm{~Hz},-\mathrm{CH}_{2} \mathrm{CH}_{2} \mathrm{CH}_{2}$ ). $0.92-$ 1.03 and $1.27-1.43$ (m. $2 \mathrm{H} .-\mathrm{CH}_{2} \mathrm{CH}_{2} \mathrm{CH}_{3}$ ). 1.11 (s. $3 \mathrm{H}$. $\left.\mathrm{C}\left(\mathrm{C}_{3}\right)_{2}\right), 1.18$ (s, $\left.3 \mathrm{H} . \mathrm{C}\left(\underline{\mathrm{CH}}_{3}\right)_{2}\right) .1 .49-1.65$ and $2.14-2.28$ (m, 2H. $\left.-\mathrm{CH}_{2} \mathrm{CH}_{2} \mathrm{CH}_{3}\right), 3.66$ (s, 3H, $\left.\mathrm{CO}_{2} \mathrm{CH}_{3}\right), 4.32$ (s. $1 \mathrm{H}$. $\mathrm{OH}), 7.22-7.44\left(\mathrm{~m}, 5 \mathrm{H}\right.$, aromatic): ${ }^{13} \mathrm{C}$ NMR $14.5\left(-\mathrm{CH}_{2}-\right.$ $\left.\left.\mathrm{CH}_{2} \mathrm{CH}_{3}\right) .169\left(-\mathrm{CH}_{2} \mathrm{CH}_{2} \mathrm{CH}_{3}\right), 21.4\left(\mathrm{C}\left(\mathrm{CH}_{3}\right)_{2}\right) .21 .8\left(\mathrm{C}_{2} \mathrm{CH}_{3}\right)_{2}\right)$. $38.1\left(-\mathrm{CH}_{2} \mathrm{CH}_{2} \mathrm{CH}_{3}\right), 50.6\left(\underline{\mathrm{C}}\left(\mathrm{CH}_{3}\right)_{2}\right), 52.0\left(\mathrm{CO}_{2} \mathrm{CH}_{3}\right) .79 .6$ 
$\left(\mathrm{C}(\mathrm{OH}) \mathrm{CH}_{2} \mathrm{CH}_{2} \mathrm{CH}_{3}\right), 126.6 .127 .1$ and $127.8\left(\mathrm{CH}_{\text {aromatic }}\right.$. $140.7(\mathrm{C}$. aromatic). 179.2 (C=O): Ir (ncal) $3600-3300$ (br. OH slreching). $1700 \mathrm{~cm}^{-1}$ (C=O streching): ClMS. $\mathrm{m} / \%$ (rcl. intensity) $251(\mathrm{M}+1,0,2), 235(0.3), 233(14), 207(3), 176$ (0.6). 150 (12). 149 (100): HRMS. $\mathrm{m} / / .251 .1647\left(\mathrm{C}_{15} \mathrm{H}_{23} \mathrm{O}_{3}\right.$ requires 251.1647 ).

41: ${ }^{1} \mathrm{H}$ NMR 0.29 (s. 9H. OSiMc $)_{3}$ ). 0.84 (t. $3 \mathrm{H}, J=6.4$ $\left.\mathrm{H} / . \mathrm{CH}_{2} \mathrm{CH}_{2} \mathrm{CH}_{3}\right) .138$ (s. $3 \mathrm{H} . \mathrm{C}\left(\mathrm{CH}_{3}\right)$ ). 1.45 (s. $3 \mathrm{H}$. $\left.\mathrm{C}\left(\mathrm{CH}_{3}\right)_{2}\right) .1 .82-1.96\left(\mathrm{~m} .2 \mathrm{H}_{0}-\mathrm{CH}_{2} \mathrm{CH}_{2} \mathrm{CH}_{3}\right) .2 .06-2.21$ (m. $2 \mathrm{H},-\mathrm{CH}_{2} \mathrm{CH}_{2} \mathrm{CH}_{3}$ ). 2.93 (s. $3 \mathrm{H}_{0} \mathrm{OCH}_{3}$ ). $7.21-7.45$ (m. $5 \mathrm{H}$. aromatic): ${ }^{3} \mathrm{C} \mathrm{NMR} 1.6\left(\mathrm{OSiMc}_{3}\right) .14 .5\left(-\mathrm{CH}_{2} \mathrm{CH}_{2} \mathrm{CH}_{3}\right) .15 .9$ $\left(-\mathrm{CH}_{2} \mathrm{CH}_{2} \mathrm{CH}_{3}\right) .24 .5\left(\mathrm{C}\left(\mathrm{CH}_{3}\right)\right) .26 .5\left(\mathrm{C}_{2}\left(\mathrm{CH}_{3}\right)\right)_{2}, 40.6\left(-\mathrm{CH}_{2}-\right.$ $\left.\mathrm{CH}_{2} \mathrm{CH}_{3}\right) .51 .3\left(\mathrm{OCH}_{3}\right) .89 .6\left(\mathrm{C}_{\left(\mathrm{CH}_{3}\right)}\right) .93 .8(\mathrm{C}(\mathrm{Ph}) \mathrm{O}-)$. $103.4\left(\mathrm{C}\left(\mathrm{OCH}_{3}\right) \mathrm{OSiMc}_{3}\right) .126 .3 .126 .6$ and $127.3(\mathrm{CH}$. aromatic). 141.9 (C. aromatic): Ir (ncal) $1250 \mathrm{~cm}^{-1}$ (C-O streching): ElMS. m/ (rel, intensity) 322 (M . 0.3). $307(0.5) .282$ (1). 265 (2). 255 (1). 237 (2). 221 (27). 175 (16). 174 (100): HRMS. $\mathrm{m} / / .322 .1972\left(\mathrm{C}_{18} \mathrm{H}_{31} \mathrm{O}_{3} \mathrm{Si}\right.$ requires 322.1964$)$.

41b: ${ }^{1} \mathrm{H}$ NMR -0.08 (s. 9H. OSiMc ). 0.82 (t. 3H. $J=6,5$ $\left.\mathrm{H} / . \mathrm{CH}_{2} \mathrm{CH}_{2} \mathrm{CH}_{3}\right) .1 .26$ (s. $3 \mathrm{H} . \mathrm{C}\left(\mathrm{CH}_{3}\right)$ ). 1.47 (s. $3 \mathrm{H}$. $\left.\mathrm{C}\left(\mathrm{CH}_{3}\right)_{2}\right)_{2} .1 .82-1.95$ (m. 2H. $\left.-\mathrm{CH}_{2} \mathrm{C}_{2} \mathrm{CH}_{3}\right) .2 .12-2.28$ (m. $2 \mathrm{H},-\mathrm{CH}_{2} \mathrm{CH}_{2} \mathrm{CH}_{3}$ ). 3.43 (s. $\left.3 \mathrm{H} . \mathrm{OCH}_{3}\right) .7 .2 \mathrm{l}-7.32$ (m. $5 \mathrm{H}$. aromatic): ${ }^{13} \mathrm{C}$ NMR $1.2\left(\mathrm{OSiMc}_{3}\right) .14 .6\left(-\mathrm{CH}_{2} \mathrm{CH}_{2} \mathrm{CH}_{3}\right)$. $\left.\left.16.1\left(-\mathrm{CH}_{2} \mathrm{CH}_{2} \mathrm{CH}_{3}\right) .25 .0\left(\mathrm{C}_{\left(\mathrm{CH}_{3}\right.}\right)_{2}\right) .26 .1\left(\mathrm{C}_{(2} \mathrm{CH}_{3}\right)_{2}\right) .40 .2$ $\left.\left(-{ }^{-} \mathrm{H}_{2} \mathrm{CH}_{2} \mathrm{CH}_{3}\right) .51 .9\left(\mathrm{OCH}_{3}\right) .89 .1\left(\mathrm{C}\left(\mathrm{CH}_{3}\right)\right)_{2}\right) .95 .2(\mathrm{C}(\mathrm{Pl}) \mathrm{O}-)$.

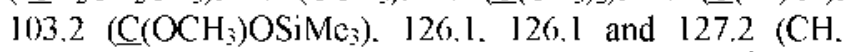
aromatic). 142.7 (C. aromatic): Ir (neat) $1250 \mathrm{~cm}^{-1}$ (C-O streching): ElMS. w// (rel, intensity) 322 (M .0.3). 307 (l). 265 (1). 221 (13). 175 (16). 174 (100): HRMS. m/. 322. 1953 $\left(\mathrm{C}_{18} \mathrm{H}_{31} \mathrm{O}_{3} \mathrm{Si}\right.$ requires 322.1964$)$.

30. ${ }^{1} \mathrm{H}$ NMR 0.29 (s. 9H. OSiMc $), 0.73$ (s. 3H. $\left.\mathrm{C}_{3}\left(\mathrm{CH}_{3}\right)_{2}\right)$. $0.81\left(\right.$ t. $3 \mathrm{H}_{.} . J=7.0 \mathrm{H} / .-\mathrm{CH}_{2} \mathrm{CH}_{2} \mathrm{CH}_{3}$ ). 1.30 (s. $\left.3 \mathrm{H} . \mathrm{C}\left(\mathrm{CH}_{3}\right)_{2}\right)$. 1.71-1.89 (m. 2H. $\left.-\mathrm{CH}_{2} \mathrm{CH}_{2} \mathrm{CH}_{3}\right), 2.06-2.2 \mathrm{l}$ (m. 2H. $-\mathrm{CH}_{2}-$ $\left.\mathrm{CH}_{2} \mathrm{CH}_{3}\right) .3 .33$ (s. $\left.3 \mathrm{H} . \mathrm{OCH}_{3}\right) .7 .2 \mathrm{l}-7.32$ (m. $5 \mathrm{H}$. aromatic): ${ }^{13} \mathrm{C} \mathrm{NMR} 1.3\left(\mathrm{OSiMc}_{3}\right)$. 14.3 $\left(-\mathrm{CH}_{2} \mathrm{CH}_{2} \mathrm{CH}_{3}\right)$. 16.7 (- $\mathrm{CH}_{2} \mathrm{CH}_{2}-$ $\left.\mathrm{CH}_{3}\right) .19 .5\left(\mathrm{C}^{2}\left(\mathrm{CH}_{3}\right)_{2}\right) .21 .7\left(\mathrm{C}\left(\mathrm{CH}_{3}\right)_{2}\right) .39 .5\left(-\mathrm{CH}_{2} \mathrm{CH}_{2} \mathrm{CH}_{3}\right)$. $50.0\left(\mathrm{OCH}_{3}\right) .51 .1\left(\mathrm{C}\left(\mathrm{CH}_{3}\right)_{2}\right) .85 .2(\mathrm{C}(\mathrm{Pl}) \mathrm{O}-) .115 .6\left(\mathrm{C}\left(\mathrm{OCH}_{3}\right)-\right.$ $\left.\mathrm{OSiMe}_{3}\right) .125 .4 .126 .1$ and $127.6(\mathrm{CH}$ aromatic). $143.0(\mathrm{C}$. aromatic): Ir (neat) $1250 \mathrm{~cm}^{-1}$ (C-O streching).

Benzene. A solution of butyrophenone (6) $(500 \mathrm{mg} .3 .4$ $\mathrm{mmol}$ ) and ketene acetal $9(1.18 \mathrm{~g} .6 .8 \mathrm{mmol})$ in $100 \mathrm{~mL}$ of benzene was irradiated for 8 h ( $c$ a. $95 \%$ conversion of 6 ). Work-up and chromatographic $(1: 8$, etlyl acetate : $n$ hexane, $v / \mathrm{s}$ ) separation (see General) gave the diastreomeric oxetanes $28 \mathrm{a}(20 \mathrm{mg}$. $2 \%$ ) and $\mathbf{2 8 b}$ ( $28 \mathrm{mg}$. 3\%), 41 a (114 $\mathrm{mg} .11 \%)$ and $41 \mathrm{~b}(30 \mathrm{mg} .3 \%) .120 \mathrm{mg}(31 \%)$ of $2.30 \mathrm{mg}$ $(6 \%)$ of $50.200 \mathrm{mg}(25 \%)$ of 18 and $73 \mathrm{mg}(7 \%)$ of 30 .

Irradiation of Valerophenone (7) and 1-Trimethylsilyloxy-1-methoxy-2,2-dimethylethylene (9). Acetonitrile. A solution of valerophenone (7) $(600 \mathrm{mg} .3 .7 \mathrm{mmol})$ and ketene acetal $9(645 \mathrm{mg} .6 .7 \mathrm{mmol})$ in $100 \mathrm{~mL}$ of acetonitrile was irradiated for $5.5 \mathrm{~h}$. (ca. $96 \%$ conversion of 7 ) Work-up and chromatographic $(1: 6$. ethyl acetate $: n$-hexane) separation (see General) gave the diastreomeric oxetanes $32 \mathrm{a}$ (12 $\mathrm{mg} .1 \%)$ and $32 \mathrm{~b}(18 \mathrm{mg} .2 \%)$. 51a (40 $\mathrm{mg} .7 \%)$ and $51 \mathrm{~b}$ (147 mg. $19 \%) .132 \mathrm{mg}(31 \%)$ of 2 and $80 \mathrm{mg}(14 \%)$ of 14 .

Benzene. A solution of valerophenone (7) $(600 \mathrm{mg} .3 .7$ mumol) and ketenc acetal $9(645 \mathrm{mg} .6 .7 \mathrm{mmol})$ in $100 \mathrm{~mL} \mathrm{of}$ ben/cne was irradiated for $6.5 \mathrm{~h}$ ( $\mathrm{ca} .88 \%$ conversion of 7 ). Work-up and chromatographic (1:6. chyl acctate:nhexane, v/v) separation (sce General) gave the diastreomeric oxetanes 32a ( $80 \mathrm{mg} .8 \%$ ) and 32b ( $12+\mathrm{mg}$. 13\%). 51 a ( 70 $\mathrm{mg} .13 \%$ ) and $51 \mathrm{~b}(140 \mathrm{mg} .19 \%) .70 \mathrm{mg}(18 \%)$ of 2 . and $58 \mathrm{mg}(11 \%)$ of 14 .

Irradiation of 2'-Acctonaphthonc (8) and 1-Trimethylsilyloxy-1-methoxy-2,2-dimethylethylenc (9). Acetonitrile. A solution of 2'-acctonaphthone (8) $(610 \mathrm{mg} .3 .6 \mathrm{mmol})$ and ketche acetal $9(1.26 \mathrm{~g} .7 .2 \mathrm{mmol})$ in $100 \mathrm{~mL}$ of acctonitrile was irradiated lor 40 h (ca. $44 \%$ conversion of 8 ). Work-up and chromatographic $(1: 1$, chy 1 acctatc $: n$-hexanc. $v / y)$ scparation (sce General) gave $48 \mathrm{mg}$ ( $11 \%$ ) ol 19.

19: ${ }^{1} \mathrm{H}$ NMR 1.21 (s. 3H. C $\left.\left(\mathrm{CH}_{3}\right)_{2}\right), 1.22$ (s. $\left.3 \mathrm{H} . \mathrm{C}\left(\mathrm{CH}_{3}\right)_{2}\right)$. 1.73 (s. $\left.3 \mathrm{H} . \mathrm{C}(\mathrm{OH}) \mathrm{CH}_{3}\right), 3.70$ (s. $\left.3 \mathrm{H}_{0}, \mathrm{OCH}_{3}\right), 4.57$ (s. $1 \mathrm{H}$. $\mathrm{OH})$. 7.45-7.94 (m. 7H. aromatic): ${ }^{13} \mathrm{C}$ NMR $21.8\left(\mathrm{C}^{(}\left(\mathrm{CH}_{3}\right)_{2}\right)$. $21.9\left(\mathrm{C}\left(\mathrm{CH}_{3}\right)_{2}\right) .25 .2\left(\mathrm{C}(\mathrm{OH}) \mathrm{CH}_{3}\right) .50 .5\left(\mathrm{OCH}_{3}\right) .52 .2$ $\left(\underline{\mathrm{C}}\left(\mathrm{CH}_{3}\right)_{2}\right) .77 .3\left(\mathrm{C}(\mathrm{OH}) \mathrm{CH}_{3}\right), 125.5,125.8,126.2,126.6$. 127.3 and $128.3(\mathrm{CH}$. aromatic). 132.3. 132.6 and 140.9 (C. aromalic). 179,0) (C=O): Ir (ncat) $3280-3600$ (br. OH stretching). $1700\left(\mathrm{C}=\mathrm{O}\right.$ stretching). $1280 \mathrm{~cm}^{-1}$ ( $\mathrm{C}-\mathrm{O}$ strecthing): EIMS. m/r (rel. intensity). $272\left(\mathrm{M}^{\prime} .2\right) .255\left(\mathrm{M}^{\prime}-\mathrm{OH} .2 \mathrm{l}\right)$. 171 (100). $155(18) .127(20)$. I02(18): HRMS. m/t.272.1 124 $\left(\mathrm{C}_{17} \mathrm{H}_{2} \mathrm{O}_{3}\right.$ requires 272,1412).

Irradiation of Acetophenone (2) and 1-(Methoxy)-1(trimethỵlsilyloxy)propenc (10). Acetonitrilc. A solution of acctophenone (2) (430 mg. $3.6 \mathrm{mmol}$ ) and ketene acctal $10(1.15 \mathrm{~g} .7 .2 \mathrm{mmol})$ in $100 \mathrm{~mL}$ of acctonitrilc was irradiated for 12 h (ca. $72 \%$ conversion of 2). Work-up and chromatographic ( $1: 15$, cthyl acctatc : $n$-hcxanc. $v / v)$ scparation (sce Gencral) gave the diastrcomeric photoproducts 20a (102 mg. $19 \%$ ) and $20 \mathrm{~b}(130 \mathrm{mg}$. $24 \%$ ). diastreomeric oxctancs $34 \mathrm{a}(60) \mathrm{mg} .8 \%$ ) of and $3+\mathrm{b}$ (115 $\mathrm{mg} .16 \%$ ). and $50 \mathrm{mg}(8 \%)$ of diol 47 .

20)a: $m p 50-52{ }^{\circ} \mathrm{C}$ : ${ }^{1} \mathrm{H}$ NMR 0.96 (d. $3 \mathrm{H} . J=7.2 \mathrm{H} \%$ $\left.\mathrm{CHCH}_{3}\right) .1 .56$ (s. $\left.3 \mathrm{H} . \mathrm{C}(\mathrm{OH}) \mathrm{CH}_{3}\right) .2 .85$ (q. $1 \mathrm{H} . J=7.2 \mathrm{H} \%$ $\mathrm{CHCH}_{3}$ ), 3.76 (s. $3 \mathrm{H}, \mathrm{OCH}_{3}$ ), 3.82 (s. $1 \mathrm{H} . \mathrm{OH}$ ). 7.23-7.46 (III. $5 \mathrm{H}$, aromatic): ${ }^{1.3} \mathrm{CNMR} 12.7\left(\mathrm{CHCH}_{3}\right), 29.9\left(\mathrm{C}(\mathrm{OH}) \mathrm{CH}_{3}\right)$. $49.3\left(\mathrm{CHCH}_{3}\right) .51 .9\left(\mathrm{OCH}_{3}\right) .74 .3\left(\underline{\mathrm{C}}(\mathrm{OH}) \mathrm{CH}_{3}\right) .124 .8$. 126.7 and 129.1 ( $\mathrm{CH}$. aromatic). 145.0 (C. aronatic). 177.6 $(\mathrm{C}=\mathrm{O})$; $\operatorname{Ir}(\mathrm{KBr}) 3300-3600$ (br. OH stretching). $1710(\mathrm{C}=\mathrm{O}$ stretching). $1200 \mathrm{~cm}^{-1}$ (C-O stretching): EIMS. $\mathrm{In} / \mathrm{z}$ (rel. intensity) 208 (1). 159 (18). 121 (100). 105 (37). 77 (15): HRMS (EI). $208.1108\left(\mathrm{C}_{12} \mathrm{H}_{16} \mathrm{O}_{3}\right.$ requires 208.1099).

20b: 'H NMR 1.32 (d. $3 \mathrm{H} . J=7.0 \mathrm{~Hz} . \mathrm{CHCH}_{3}$ ). 1.46 (s. $\left.3 \mathrm{H} . \mathrm{C}(\mathrm{OH}) \mathrm{CH}_{3}\right) .3 .03$ (q. $\left.1 \mathrm{H} . J=7.0 \mathrm{~Hz} . \mathrm{CHCH}_{3}\right) .3 .46$ (s. $\left.3 \mathrm{H} . \mathrm{OCH}_{3}\right) .4 .03$ (s. $1 \mathrm{H} . \mathrm{OH}$ ). $7.21-7.45$ (m. $5 \mathrm{H}$. aromatic): ${ }^{1.7} \mathrm{C} \mathrm{NMR} 12.4\left(\mathrm{CHCH}_{3}\right) .26 .6\left(\mathrm{C}(\mathrm{OH}) \mathrm{CH}_{3}\right) .48 .5\left(\mathrm{CHCH}_{3}\right)$. $51.6\left(\mathrm{OCH}_{3}\right) .74 .6\left(\underline{\mathrm{C}}(\mathrm{OH}) \mathrm{CH}_{3}\right) .124 .6 .126 .7$ and 128.1 $(\mathrm{CH}$. aromatic), $147.5(\mathrm{C}$, aronatic), $177.0(\mathrm{C}=\mathrm{O})$ : Ir (neat) 3300-3600 (br. OH stretching). 1710 ( $\mathrm{C}=\mathrm{O}$ stretching). 1200 $\mathrm{cm}^{-1}$ (C-O stretching): EIMS, $\mathrm{m} / \mathrm{z}$ (rel. intensity) $208(0.3)$. 191 (2), 159 (6), 121 (100), 105 (23). 88 (11), 77 (14): HRMS (EI). $208.1100\left(\mathrm{C}_{13} \mathrm{H}_{66} \mathrm{O}_{3}\right.$ requires 208.1099$)$.

34a: 'H NMR 0.20 (s. 9H. OSiMe $) .1 .43$ (d. 3H. $J=6.3$ Hz. $\mathrm{CHC}_{2}$ ). 1.77 (s. $\left.3 \mathrm{H}, \mathrm{C}(\mathrm{Pl}) \mathrm{CH}_{3}\right) .2 .58$ (s. $\left.3 \mathrm{H} . \mathrm{OCH}_{3}\right)$. 
4.87 (q. $\left.\mathrm{lH}, J=6.3 \mathrm{H} / . \mathrm{CHCH}_{3}\right), 7.27-7.59$ ) (m. $5 \mathrm{H}$ aromatic): ${ }^{13} \mathrm{C}$ NMR $1.1\left(\mathrm{OSiMc}_{3}\right) .15 .4\left(\mathrm{CH}^{\mathrm{CH}} \mathrm{H}_{3}\right) .23 .3\left(\mathrm{C}(\mathrm{Ph}) \mathrm{CH}_{3}\right)$. $49.4\left(\mathrm{OCH}_{3}\right) .84 .6\left(\mathrm{CHCH}_{3}\right) .93 .0\left(\mathrm{C}(\mathrm{Ph}) \mathrm{CH}_{3}\right) .101 .2\left(\mathrm{C}_{(\mathrm{OCH}}\right)-$ $\left.\mathrm{OSiMc}_{3}\right), 126.8,127.2$ and $127.6(\mathrm{CH}$ aromatic). 142.1 (C. aromatic): Ir (neat) $1220 \mathrm{~cm}^{-1}$ (C-O stretching): EIMS, $\mathrm{m} / \%$ (rel. intensity) 280 (0.8), 279 (3). 236 (12). 193 (100). 159 (85). 132 (29). 105 (19): HRMS (EJ). $280.1485\left(\mathrm{C}_{15} \mathrm{H}_{24} \mathrm{O}_{3} \mathrm{Si}\right.$ requires $280 \mathrm{l} 495$ ).

34b: ${ }^{1} \mathrm{H}$ NMR -0.22 (s. 9H. OSiMc 3 ). 1.35 (d. $3 \mathrm{H}_{.} . J=6.3$ $\left.\mathrm{H} / . . \mathrm{CHCH}_{3}\right) .1 .75$ (s. $3 \mathrm{H}_{0} \mathrm{C}(\mathrm{Ph}) \mathrm{CH}_{3}$ ). 3.31 (s. $3 \mathrm{H}_{.} \mathrm{OCH}_{3}$ ). 4.91 (q. lH. $\left.J=6.3 \mathrm{H} z . \mathrm{CHCH}_{3}\right) .7 .21-7.48(\mathrm{~m} .5 \mathrm{H}$, aromatic): ${ }^{13} \mathrm{C}$ NMR 1.1 (OSiMc 3$)$. $16.7\left(\mathrm{CHCH}_{3}\right) .23 .4$ $\left(\mathrm{C}(\mathrm{Ph}) \mathrm{CH}_{3}\right) .51 .1\left(\mathrm{OCH}_{3}\right) .82 .3\left(\mathrm{CHCH}_{3}\right) .93 .3\left(\mathrm{C}(\mathrm{Ph}) \mathrm{CH}_{3}\right)$. $103.1\left(\mathrm{C}\left(\mathrm{OCH}_{3}\right) \mathrm{OSiMc}_{3}\right) .126 .8 .127 .1$ and $127.6(\mathrm{CH}$. aromatic). 142.7 (C. aromatic): Ir(ncat) $1220 \mathrm{~cm}^{-1}$ (C-O stretching): ElMS. m/s (rel, intensity) $280(0.04) .237$ (2). 221 (1). $193(46) .160(44) .105(53) .73$ (100): HRMS (EI). $280.1483\left(\mathrm{C}_{15} \mathrm{H}_{1+} \mathrm{O}_{3} \mathrm{Si}\right.$ requires 280.1495$)$.

Benzene. A solution of acclophenone (2) (430 mg. 3.6 mmol) and ketenc acetal 10 (1.15 g. $7.2 \mathrm{mmol})$ in $100 \mathrm{~mL}$ of benzene was irradiated for 14 he (ca. $47 \%$ conversion of 2 ). Work-up and chromatographic ( $1: 15$. ethyl acctate: $n$ hexanc. $v / \mathrm{s}$ ) separation (sce Gencral) gave $53 \mathrm{mg}(15 \%)$ of 20a. $88 \mathrm{mg}(25 \%)$ of $20 \mathrm{~b}$. $100 \mathrm{mg}(21 \%)$ of $34 a$ and $160 \mathrm{mg}$ $(34 \%)$ or $34 \mathbf{b}$.

Irradiation of Benzophenone (3) and 1-(Methoxy)-1(trimethylsilyloxy) propene (10). Acetonitrilc. A solution of ben $\%$ ophenone (3) $(656 \mathrm{mg} .3 .6 \mathrm{mmol})$ and ketene acclal 10) $(1.15 \mathrm{~g} .7 .2 \mathrm{mmol})$ in $100 \mathrm{~mL}$ of acctonitrilc was irradiated for 8 b (ca. $85 \%$ conversion of 3 ). Work-up and chromatographic (1: 10. cthyl acctate: $n$-hexanc. $y / v)$ separation (sce General) gave the diastrcomeric oxclanes 37a (122 mg. $12 \%$ ) of and $37 \mathrm{~b}(260 \mathrm{mg} .25 \%$ ). $340 \mathrm{mg}(41 \%)$ of 21 . and $34 \mathrm{mg}(6 \%)$ of 48 .

21: $\mathrm{mp} 128-1300^{\circ} \mathrm{C}$ : ${ }^{~} \mathrm{H}$ NMR 1.23 (d. $3 \mathrm{H} . J=7.2 \mathrm{H} \%$ $\mathrm{CHCH}_{3}$ ). 3.63 (s. $3 \mathrm{H} . \mathrm{OCH}_{3}$ ). 3.74 (q.l $\mathrm{H}, J=7.2 \mathrm{H} \%$ $\left.\mathrm{CHCH}_{3}\right) .477$ (s. lH. OH). 7.16-7.64 (m. 10H. aromatic): ${ }^{13} \mathrm{C}$ NMR $12.9\left(\mathrm{CHCH}_{3}\right) .46 .7\left(\mathrm{CO}_{2} \mathrm{CH}_{3}\right) .51 .9\left(\mathrm{CHCH}_{3}\right)$. $78.0\left(\underline{\mathrm{C}}(\mathrm{Ph})_{2} \mathrm{OH}\right) .125 .2,125.3,126.5 .126 .9,128.1$ and 128.3 (CH. aromatic). 144.0 and 147.5 (C. aromatic). 177.9 $\left(\mathrm{C}=\mathrm{O}\right.$ ): Ir (neat), 3200-3600 (br. OH streching). $1710 \mathrm{~cm}^{-1}$ $\left(\mathrm{C}=\mathrm{O}\right.$ streching): EIMS $\mathrm{m} / \mathrm{z}$ (rel. intensity) $270\left(\mathrm{M}^{-}, 0.2\right)$. 239 (1). 183 (100). 165 (2). 105 (65) .77 (31): HRMS, $\mathrm{m} / \mathrm{z}$ $270.1263\left(\mathrm{C}_{17} \mathrm{H}_{3} \mathrm{O}_{3}\right.$ requires 270.1256$)$.

37a: ${ }^{1}$ H NMR -0.06 (s, 9H. OSiMe $), 1.43$ (d. 3H. $J=6.2$ $\mathrm{Hz}, \mathrm{CHC} \underline{H}_{3}$ ). 3.08 (s. $3 \mathrm{H} . \mathrm{OCH}_{3}$ ). 4.92 (q. $1 \mathrm{H} . J=6.2 \mathrm{~Hz}$. $\left.\mathrm{C}^{\mathrm{H}} \mathrm{CH}_{3}\right) .7 .20-7.65$ (m. $10 \mathrm{H}$. aromatic); ${ }^{13} \mathrm{C}$ NMR 1.2 (OSiMe $) .16 .7\left(\mathrm{CHCH}_{3}\right) .51 .4\left(\mathrm{OCH}_{3}\right) .82 .6\left(\mathrm{CHCH}_{3}\right) .95 .8$ $(\underline{\mathrm{C}}(\mathrm{Ph}) 2), 103.8\left(\underline{\mathrm{C}}\left(\mathrm{OCH}_{3}\right) \mathrm{OSSiMe}_{3}\right), 126.6,127.0 .127 .2$. 127.3. 127.9 and 128.2 (CH. aromatic) 141.3 and 141.5 (C. aromatic); Ir (neat). $1250 \mathrm{~cm}^{-1}$ (C-O streching): CIMS. $\mathrm{m} / \mathrm{z}$ (rel. intensity) 343 (6). 255 (53). 183 (33) 160 (98). 105 (100). 73 (94): $\mathrm{HRMS}(\mathrm{CI}) 34.1734\left(\mathrm{C}_{3 \mathrm{i}} \mathrm{H}_{2} \mathrm{O}_{3} \mathrm{Si}\right.$ requires 3+3.1729).

37b: ${ }^{1} \mathrm{H}$ NMR 0.01 (s. 9H. OSiMe $) .1 .58$ (d. 3H. $J=6.3$ $\mathrm{Hz}, \mathrm{CHC} \underline{H}_{3}$ ). 2.90 (s. $3 \mathrm{H} . \mathrm{OCH}_{3}$ ). 4.81 (q. $1 \mathrm{H} . J=6.2 \mathrm{~Hz}$. $\mathrm{C}_{\mathrm{HCH}}$ ). 7.26-7.69 (m. $10 \mathrm{H}$. aromatic): ${ }^{1.3} \mathrm{C}$ NMR 0.7
$\left(\mathrm{OSiMe}_{3}\right), 15.2\left(\mathrm{CHCH}_{3}\right), 49.7\left(\mathrm{OCH}_{3}\right) .84 .5\left(\mathrm{CHCH}_{3}\right) .95 .9$ $\left(\underline{\mathrm{C}}(\mathrm{Ph})_{2}\right), 101.8\left(\underline{\mathrm{C}}\left(\mathrm{OCH}_{3}\right) \mathrm{OSSiMe}_{3}\right), 126,7,127.1,127.2$. 127.4 .127 .6 and $127.9(\mathrm{CH}$. aromatic). 140.8 and $141.2(\mathrm{C}$. aromatic): Ir (neat). $1250 \mathrm{~cm}^{-1}$ (C-O strching): CIMS, $\mathrm{m} / \mathrm{s}$ (rel. intensity) $343\left(\mathrm{M}^{\prime}+1.1\right) .255$ (3). 225 (3). 210 (20). 198 (19). $197(100) .183(45) .166(42)$ : HRMS. m/ 343.1725 $\left(\mathrm{C}_{30} \mathrm{H}_{27} \mathrm{O}_{3} \mathrm{Si}\right.$ requires 343.1729$)$.

Benzenc. A solution of ben/ophenone (3) $(656 \mathrm{mg} .3 .6$ mmol) and ketenc acctal $10(1.15 \mathrm{~g} .7 .2 \mathrm{mmol})$ in $100 \mathrm{~mL}$ of acetonitrile was irradiated for $7 \mathrm{~h}(c a .90 \%$ conversion of 3 ). Work-up and chromatographic ( $1: 10$. ethyl acctate: nhexanc. $v / v)$ scparation (sce Gencral) gave $380 \mathrm{mg}(39 \%)$ of 21. $147 \mathrm{mg}(12 \%)$ of $37 \mathrm{a} .294 \mathrm{mg}(24 \%)$ of $37 \mathrm{~b}$ and $44 \mathrm{mg}$ (14\%) of 48 .

Irradiation of 4-Methoxyacetophenone (4) and 1(Methoxy)-1-(trimethylsilyloxy) propene (10). Acetonitrilc. A solution of 4-methoxyacetophenone (4) $(540 \mathrm{mg}$. $3.6 \mathrm{mmol}$ ) and ketcne acctal $10(1.15 \mathrm{~g} .7 .2 \mathrm{mmol})$ in 100 $\mathrm{mL}$ of acctonitrilc was irradiated for 50 h (ca. $26 \%$ conversion of 4). Work-up and chromatographic ( $1:$ t. chy 1 acctate : $n$-hexanc) separation (sec Gencral) gave the diastrcomeric photoproducts 22a (38 mg. 17\%) and 22b (40 mg. $18 \%$ ). diastrcomeric oxctancs $35 a(70 \mathrm{mg} .26 \%$ ) of and $35 b(60 \mathrm{mg}$. 22\%).

22a: $\mathrm{mp} 45-47^{\circ} \mathrm{C}$ : ${ }^{1} \mathrm{H}$ NMR 0.97 (d. $3 \mathrm{H} . J=7.2 \mathrm{H} \%$ $\left.\mathrm{CHCH}_{3}\right) .1 .54$ (s. $\left.3 \mathrm{H} . \mathrm{C}(\mathrm{OH}) \mathrm{CH}_{3}\right) .2 .56$ (s. IH. OH). $2.8 \mathrm{I}$ (q. $\mathrm{l}$ H. $J=7.2 \mathrm{H} \% \mathrm{CHCH}_{3}$ ). 3.75 (s. $3 \mathrm{H} . \mathrm{CO}_{2} \mathrm{CH}_{3}$ ). 3.80 (s. $3 \mathrm{H} . \mathrm{Ph}-\mathrm{OCH}_{3}$ ). 6.87 (d. $2 \mathrm{H} . J=9.0 \mathrm{H} z$. aromatic). 7.36 (d. $2 \mathrm{H}, J=9.0 \mathrm{H} \%$ aromatic): ${ }^{13} \mathrm{C}$ NMR $12.7\left(\mathrm{CHCH}_{3}\right) .29 .8$ $\left(\mathrm{C}(\mathrm{OH}) \mathrm{CH}_{3}\right) .49 .4\left(\mathrm{CO}_{2} \mathrm{CH}_{3}\right) .51 .8\left(\mathrm{CHCH}_{3}\right) .55 .1(\mathrm{Ph}-$ $\left.\mathrm{OCH}_{3}\right) .74 .0\left(\mathrm{C}(\mathrm{OH}) \mathrm{CH}_{3}\right) .113 .6$ and $126.0(\mathrm{CH}$. aromatic). 137.1 and $158.2(\mathrm{C}$ aromatic). $177.6(\mathrm{C}=\mathrm{O})$ : $\operatorname{Ir}(\mathrm{KBr}) 3600$ 3200 (br. $\mathrm{OH}$ streching). $1700 \mathrm{~cm}^{-1}(\mathrm{C}=\mathrm{O}$ streching): EIMS. $\mathrm{m} / \mathrm{s}$ (rcl, intensity) 238 (M'. 2). 22l (4). $\mathrm{l} 6 \mathrm{l}$ (1). 152 (ll). 151 (I00). $135(13)$ : HRMS. m/. 238.1209 $\left(\mathrm{C}_{13} \mathrm{H}_{18} \mathrm{O}_{4}\right.$ requires 238.1205 ).

22b: 'H NMR 1.30 (d. $3 \mathrm{H}_{.} . J=7.2 \mathrm{H} \% . \mathrm{CHCH}_{3}$ ). $\mathrm{l} .44$ (s. $\left.3 \mathrm{H} . \mathrm{C}(\mathrm{OH}) \mathrm{CH}_{3}\right) .2 .99$ (q. $\left.1 \mathrm{H} . J=7.2 \mathrm{~Hz} . \mathrm{CHCH}_{3}\right) .3 .49$ (s. $3 \mathrm{H} . \mathrm{CO}_{2} \mathrm{CH}_{3}$ ). 3.79 (s. $3 \mathrm{H} . \mathrm{Ph}-\mathrm{OC} \underline{H}_{3}$ ). 3.97 (s. $1 \mathrm{H} . \mathrm{OH}$ ). 6.85 (d. $2 \mathrm{H} . J=9.0 \mathrm{~Hz}$ aromatic). 7.34 (d. $2 \mathrm{H} . J=9.0 \mathrm{~Hz}$. aromatic): ${ }^{13} \mathrm{C}$ NMR $12.5\left(\mathrm{CHCH}_{3}\right), 26.6\left(\mathrm{C}(\mathrm{OH}) \mathrm{CH}_{3}\right) .48 .7$ $\left(\mathrm{CO}_{2} \mathrm{CH}_{3}\right) .51 .6\left(\mathrm{CHCH}_{3}\right) .55 .1\left(\mathrm{Ph}_{-} \mathrm{OCH}_{3}\right) .74 .4\left(\underline{\mathrm{C}}(\mathrm{OH}) \mathrm{CH}_{3}\right)$. 113.4 and $125.8(\mathrm{CH}$. aromatic). 139.6 and $158.3(\mathrm{C}$. aromatic). 177.1 ( $\mathrm{C}=\mathrm{O})$ : Ir (neat). 3600-3200 (br. OH streching). $1710 \mathrm{~cm}^{-1}(\mathrm{C}=\mathrm{O}$ streching): EIMS. $\mathrm{nl} / \mathrm{z}$ (rel. intensity) $238\left(\mathrm{M}^{-}, 2\right) .221$ (2). 161 (3). 160 (5). 159 (12). $151(100) .1+9(14)$ : HRMS. $\mathrm{nl} / \mathrm{z} 238.1206\left(\mathrm{C}_{13} \mathrm{H}_{18} \mathrm{O}_{4}\right.$ requires 238.1205 ).

35a: ${ }^{\prime} \mathrm{H}$ NMR 0.19 (s. 9H. OSiMe $) .1 .42$ (d. $3 \mathrm{H} . J=6.8$ Hz. $\mathrm{CHCH}_{3}$ ). 1.74 (s. $3 \mathrm{H}, \mathrm{C}\left(\mathrm{Ph}-\mathrm{OCH}_{3}\right) \underline{\mathrm{H}}_{3}$ ). 2.57 (s. $3 \mathrm{H}$. $\left.\mathrm{C}\left(\mathrm{OSiMe}_{3}\right) \mathrm{OCH}_{3}\right) 3.82$ (s. $\left.3 \mathrm{H} . \mathrm{Ph}-\mathrm{OC} \underline{H}_{3}\right) .4 .84$ (q. $1 \mathrm{H} . J=$ $6.3 \mathrm{~Hz} . \mathrm{CHCH}_{3}$ ). 6.88 (d. $2 \mathrm{H} . J=8.4 \mathrm{~Hz}$ aronatic) 7.47 (d. $2 \mathrm{H} . J=8.4 \mathrm{~Hz}$. aromatic): ${ }^{13} \mathrm{C}$ NMR $1.1\left(\mathrm{OSSMe}_{3}\right), 15.3$ $\left(\mathrm{CHCH}_{3}\right) .23 .1\left(\mathrm{C}\left(\mathrm{Pll}_{1}-\mathrm{OCH}_{3}\right) \mathrm{CH}_{3}\right) .49 .3\left(\mathrm{C}\left(\mathrm{OSSiMe}_{3}\right) \mathrm{OCH}_{3}\right)$. $55.1\left(\mathrm{Ph}-\mathrm{OCH}_{3}\right) .84 .3\left(\mathrm{CHCH}_{3}\right) .92 .6\left(\mathrm{C}\left(\mathrm{Ph}-\mathrm{OCH}_{3}\right) \mathrm{CH}_{3}\right)$. 101.2 ( $\left.\mathrm{C}\left(\mathrm{OSiMe}_{3}\right) \mathrm{OCH}_{3}\right), 112.9$ and 128.1 (CH. aronatic). 134.2 and 158.9 (C. aromatic): Ir (neat) $1250 \mathrm{~cm}^{-1}$ (C-O 
streching): CIMS $\mathrm{m} / \mathrm{t}$ (rel. intensity) $295\left(\mathrm{M}^{\prime}+\mathrm{l}, \mathrm{l}\right), 267$ (10), 224 (11), $223(60), 177$ (3), 162 (29), 160 (100): HRMS $\mathrm{m} / .294 .1656\left(\mathrm{C}_{16} \mathrm{H}_{26} \mathrm{O}_{3} \mathrm{Si}\right.$ requires $\left.294.165 \mathrm{l}\right)$.

35b: ${ }^{1} \mathrm{H}$ NMR $-0.2 \mathrm{l}$ (s. 9H. OSiMe 3 ), 1.35 (d. $3 \mathrm{H}, J=6.3$ $\mathrm{H} / . \mathrm{CHCH}_{3}$ ). 1.73 (s. 3H. $\mathrm{C}\left(\mathrm{Ph}-\mathrm{OCH}_{3}\right) \mathrm{CH}_{3}$ ). 3.29 (s. $3 \mathrm{H}$. $\left.\mathrm{C}\left(\mathrm{OSiMc}_{3}\right) \mathrm{OCH}_{3}\right) .3 .81$ (s. $\left.3 \mathrm{H} . \mathrm{Ph}_{2}-\mathrm{OCH}_{3}\right) .4 .89$ (q. $1 \mathrm{H}_{.} J=$ $6.2 \mathrm{H} / . \mathrm{CHCH}_{3}$ ). 6.87 (d. $2 \mathrm{H} . J=8.9 \mathrm{H} / .$. aromatic). 7.38 (d. $2 \mathrm{H} . J=8.8 \mathrm{H} / \ldots$ aromatic): ${ }^{13} \mathrm{C}$ NMR $1.1\left(\mathrm{OSiMc}_{3}\right) .16 .5$ $\left(\mathrm{CHCH}_{3}\right) .23 .1\left(\mathrm{C}\left(\mathrm{Ph}_{1}-\mathrm{OCH}_{3}\right) \mathrm{CH}_{3}\right) .51 .0\left(\mathrm{C}\left(\mathrm{OSiMc}_{3}\right) \mathrm{OCH}_{3}\right)$. $55.2\left(\mathrm{Ph}_{-}-\mathrm{OCH}_{3}\right) .81 .9\left(\mathrm{CHCH}_{3}\right) .92 .9\left(\mathrm{C}\left(\mathrm{Ph}_{2}-\mathrm{OCH}_{3}\right) \mathrm{CH}_{3}\right)$. $103.3\left(\mathrm{C}\left(\mathrm{OSiMc}_{3}\right) \mathrm{OCH}_{3}\right) .112 .9$ and $128.4\left(\mathrm{CH}_{\text {. aromatic }}\right)$. 134.8 and 158.9 (C. aromatic): Ir (neat) $1250 \mathrm{~cm}^{-1}$ (C-O streching): CIMS $\mathrm{m} / \mathrm{t}$ (rel. intensity) $295\left(\mathrm{M}^{\prime}+\mathrm{l} . \mathrm{l}\right) .266$ (16). 251 (3). 223 (16). 162 (29). 160 (100): HRMS. m/t. $294.1659\left(\mathrm{C}_{16} \mathrm{H}_{26} \mathrm{O}_{3} \mathrm{Si}\right.$ requires 294.1651$)$.

Benzene. A solution of 4-methoxyacetophenone (4) (540) $\mathrm{mg} .3 .6 \mathrm{mmol})$ and ketene acetal $10(1.15 \mathrm{~g} .7 .2 \mathrm{mmol})$ in $100 \mathrm{~mL}$ of benzene was irradiated for $19 \mathrm{~h}$ (ca. $46 \%$ conversion of 4$)$. Work-up and chromatographic $(1: 4$. cthy 1 acetatc : $n$-hexane. $/ /$ ) separation (see Gencral) gave $95 \mathrm{mg}$ (24\%) of $22 \mathrm{a} .122 \mathrm{mg}(31 \%)$ of $22 \mathrm{~b} .88 \mathrm{mg}(18 \%)$ of $35 \mathrm{a}$ and $120 \mathrm{mg}(25 \%)$ of $35 \mathrm{~b}$.

Irradiation of 4-Cyanoacetophenone (5) and 1-(Methoxy)-1-(trimethylsilyloxy) propene (10). Benzenc. A solution of 4-cyanoacclophenone (5) (520 $\mathrm{mg} .3 .6 \mathrm{mmol}$ ) and kelene acctal 10 (1.15 g. 7.2 mmol) in $100 \mathrm{~mL}$ of ben/ene was irradiated for 13.5 h (ca. $87 \%$ conversion ol 5 ). Work-up and chromatographic $(1: 5$. chlyl acclate $: n$-hexanc. $v / y)$ separation (see General) gave the diastreomeric photoproducts 23a (124 mg. $17 \%$ ) and 23b (340 mg. 47\%). diastrcomcric oxctanes $36 \mathrm{a}(78 \mathrm{mg} .8 \%$ ) of and $36 \mathrm{~b}(160 \mathrm{mg} .17 \%)$. and $45 \mathrm{mg}(5 \%)$ of diol 49.

23a: $m p$ 84-86 ${ }^{\circ} \mathrm{C}$. ${ }^{1} \mathrm{H}$ NMR 0.92 (d. $3 \mathrm{H}, J=7.1 \mathrm{H} \%$ $\left.\mathrm{CHCH}_{3}\right) .1 .54$ (s. $\left.3 \mathrm{H} . \mathrm{C}(\mathrm{OH}) \mathrm{CH}_{3}\right) .2 .83$ (q. $1 \mathrm{H} . J=7.1 \mathrm{H} \%$ $\mathrm{CHCH}_{3}$ ). 3.77 (s. $3 \mathrm{H} . \mathrm{CO}_{2} \mathrm{CH}_{3}$ ). 4.03 (s. IH. OH). 7.52-7.66 (mi. $4 \mathrm{H}$. aromatic): ${ }^{13} \mathrm{C} \mathrm{NMR} 12.6\left(\mathrm{CHCH}_{3}\right) .29 .5\left(\mathrm{C}(\mathrm{OH}) \underline{\mathrm{C}} \mathrm{H}_{3}\right)$. $48.7\left(\mathrm{CO}_{2} \mathrm{CH}_{3}\right) .52 .0\left(\mathrm{CHCH}_{3}\right) .74 .2\left(\underline{\mathrm{C}}(\mathrm{OH}) \mathrm{CH}_{3}\right) .110 .6$ (CN). 126.8 and $131.9(\mathrm{CH}$ aromatic). 118.9 and $150.4(\mathrm{C}$. aromatic) $177.1(\mathrm{C}=\mathrm{O})$; $\mathrm{Ir}(\mathrm{KBr}) .3600-3200$ (br. OH streching). 2230 ( $\mathrm{CN}$ streching), $1710 \mathrm{~cm}^{-1}(\mathrm{C}=\mathrm{O}$ streching): CIMS $\mathrm{m} / \mathrm{z}$ (rel. intensity) $234\left(\mathrm{M}^{-}+1,4\right) .218(8) .146(49)$. $130(46), 122(8) .116(4), 102(17), 88$ (100): HRMS. m/z $234.1136\left(\mathrm{C}_{1} \mathrm{H}_{16} \mathrm{NO}_{3}\right.$ requires 234.1130$)$.

23b: $m p$ 36-38'C. 'H NMR 1.36 (d. $3 \mathrm{H} . J=7.0 \mathrm{~Hz}$. $\left.\mathrm{CHCH}_{3}\right) .1 .44$ (s. $\left.3 \mathrm{H} . \mathrm{C}(\mathrm{OH}) \underline{H}_{3}\right) .3 .01(\mathrm{q} .1 \mathrm{H} . J=7.0 \mathrm{~Hz}$. $\mathrm{CHCH}_{3}$ ). 3.47 (s. $3 \mathrm{H} . \mathrm{CO}_{2} \mathrm{CH}_{3}$ ). 4.19 (s. $1 \mathrm{H} . \mathrm{OH}$ ). $7.52-7.65$ (Im. 4H, aromatic): ${ }^{13} \mathrm{C}$ NMR $12.2(\mathrm{CHCH} 3), 26.5(\mathrm{C}(\mathrm{OH})-$ $\left.\mathrm{CH}_{3}\right) .47 .9\left(\mathrm{CO}_{2} \mathrm{CH}_{3}\right) .51 .7\left(\mathrm{CHCH}_{3}\right) .74 .5\left(\underline{\mathrm{C}}(\mathrm{OH}) \mathrm{CH}_{3}\right)$. $110.6(\mathrm{CN}) .125 .5$ and $131.9(\mathrm{CH}$ aromatic). 118.6 and 153.0 (C. aromatic). $176.6(\mathrm{C}=\mathrm{O})$ : $\operatorname{Ir}(\mathrm{KBr}) .3600-3200(\mathrm{br}$. $\mathrm{OH}$ streching). 2230 (CN streching). $1710 \mathrm{~cm}^{-1}$ (C=O streching): CIMS $\mathrm{m} / \mathrm{z}$ (rel. intensity) $234\left(\mathrm{M}^{+}+1,5\right), 216$ (8). $146(51), 130$ (22). 102 (8). 88 (100). 57 (22): HRMS. $\mathrm{m} / \mathrm{z} 234.1137\left(\mathrm{C}_{13} \mathrm{H}_{16} \mathrm{NO}_{3}\right.$ requires 234.1130$)$.

36a: 'H NMR 0.2l (s. 9H. OSiMe 3 ). 1.41 (d. 3H. $J=6.3$ $\mathrm{Hz}, \mathrm{CHCH}_{3}$ ) 1.73 (s. $3 \mathrm{H}, \mathrm{C}(\mathrm{Ph}-\mathrm{CN}) \mathrm{CH}_{3}$ ). 2.63 (s. $3 \mathrm{H}$. $\mathrm{OCH}_{3}$ ). 4.88 (q. $1 \mathrm{H} . J=6.3 \mathrm{~Hz}, \mathrm{CHCH}_{3}$ ). 7.64 (s. $4 \mathrm{H}$. aromatic): ${ }^{13} \mathrm{C}$ NMR $1.1\left(\mathrm{OSiMe}_{3}\right), 15.5\left(\mathrm{CHCH}_{3}\right), 23.2$ $\left(\mathrm{C}(\mathrm{Ph}-\mathrm{CN}) \mathrm{CH}_{3}\right), 49.6\left(\mathrm{OCH}_{3}\right), 85.2\left(\mathrm{CHCH}_{3}\right) .92 .7(\mathrm{C}(\mathrm{Ph}-$ $\left.\mathrm{CN} \mathrm{CH}_{3}\right) .101 .9\left(\mathrm{C}\left(\mathrm{OSiMe}_{3}\right) \mathrm{OCH}_{3}\right) .110 .9(\mathrm{CN}) .127 .4$ and 131.4 ( $\mathrm{CH}$. aromatic), 147.8 (C.aromatic): Ir (ncat) 2230) (CN streching): CIMS. $\mathrm{m} / \mathrm{t}$ (rcl. intensity) $306\left(\mathrm{M}^{\prime}+1.0 .1\right)$. 290 (1). 261 (7). $248(3) .218$ (40). $186(5) .105(38) .89$ (35). 73 (100): HRMS. $\mathrm{m} / \mathrm{s} .306 .1536\left(\mathrm{C}_{16} \mathrm{H}_{24} \mathrm{NO}_{3} \mathrm{Si}\right.$ requires $306.1526)$

36): ${ }^{1} \mathrm{H}$ NMR $-(0.18$ (s. 9H. OSiMc ). 1.31 (d. 3 H. $J=6.3$ $\left.\mathrm{H} \% \mathrm{CHCH}_{3}\right) .1 .71$ (s. $3 \mathrm{H}, \mathrm{C}\left(\mathrm{Ph}_{-}-\mathrm{CN}\right) \mathrm{CH}_{3}$ ). 3.34 (s. $3 \mathrm{H}$. $\left.\mathrm{OCH}_{3}\right) .4 .92\left(\mathrm{q} . \mathrm{lH} . J=6.3 \mathrm{H} / . \mathrm{CHCH}_{3}\right) .7 .5 \mathrm{l}-7.66(\mathrm{~m} .+\mathrm{H}$. aromatic): ${ }^{13} \mathrm{C}$ NMR $1.1\left(\mathrm{OSiMc}_{3}\right), 16.8\left(\mathrm{CHCH}_{3}\right), 23.3$ $\left(\mathrm{C}(\mathrm{Ph}-\mathrm{CN}) \mathrm{CH}_{3}\right), 51.3\left(\mathrm{OCH}_{3}\right), 82.8\left(\mathrm{CHCH}_{3}\right) .93 .0(\mathrm{C}(\mathrm{Ph}-$ $\left.\mathrm{CN}) \mathrm{CH}_{3}\right) .102 .8\left(\mathrm{C}\left(\mathrm{OSiMc}_{3}\right) \mathrm{OCH}_{3}\right) .110 .7(\mathrm{CN}) .127 .2$ and $131.3(\mathrm{CH}$. aromatic) 148.5 (C.aromatic): Ir (neat) 2230 (CN streching): CIMS. m/ (rcl. intensity) $306\left(\mathrm{M}^{\prime}+1,0.04\right) .290$ (1). 26] (7). 248 (4). 218 (26). $160(94) .105$ (68). 73 (100): HRMS. $\mathrm{m} / \mathrm{s} 306.1527\left(\mathrm{C}_{66} \mathrm{H}_{24} \mathrm{NO}_{3} \mathrm{Si}\right.$ requires 306.1526$)$.

Irradiation of Acctophenone (2) and 1-(Methoxy)-1(trimethylsilyloxy)ethanc (11). Acetonitrile. A solution of acctophenonc (2) (430 $\mathrm{mg} .3 .6 \mathrm{mmol})$ and ketene acctal 11 (1.05 g. $7.2 \mathrm{mmol}$ ) in $100 \mathrm{~mL}$ of acctonitrilc was irradiated for 20 h (ca. $49 \%$ conversion of 2 ). Work-up and chromatographic ( $1: 4$. cthyl acctate: $n$-hexanc. $v / v)$ separation (sec Gencral) gave the diastrcomeric oxetancs 38 a ( $75 \mathrm{mg}$. $16 \%$ ) and $38 \mathrm{~b}(140 \mathrm{mg} .30 \%$ ). and $86 \mathrm{mg}(25 \%)$ of 24 .

24: ${ }^{\prime} \mathrm{H}$ NMR 1.56 (s. $\left.3 \mathrm{H} . \mathrm{C}(\mathrm{OH}) \mathrm{CH}_{3}\right) .2 .8 \mathrm{l}(\mathrm{d} . \mathrm{lH} . J=16$ $\mathrm{H} / . \mathrm{CH}_{2}$ ). $3.0 \mathrm{l}$ (d. $\mathrm{IH} . J=16 \mathrm{H} \% \mathrm{CH}_{2}$ ). $3.6 \mathrm{l}$ (s. $3 \mathrm{H}$. $\mathrm{CO}_{2} \mathrm{CH}_{3}$ ). 4.34 (s. IH. OH). 7.24-7.48 (m. 5H. aromatic): ${ }^{13} \mathrm{C}$ NMR $30.5\left(\mathrm{C}(\mathrm{OH}) \mathrm{CH}_{3}\right) .46 .1\left(\mathrm{CH}_{2}\right) .51 .6\left(\mathrm{CO}_{2} \mathrm{CH}_{3}\right)$. $72.6\left(\mathrm{C}(\mathrm{OH}) \mathrm{CH}_{3}\right) .124,3.126,8$ and $128.5(\mathrm{CH}$. aromatic). 146.8 (C. aromatic). 173.0 ( $\mathrm{C}=\mathrm{O})$ : Ir (ncat) $3600(3200$ (br. $\mathrm{OH}$ strcching). $1720 \mathrm{~cm}^{-1}(\mathrm{C}=\mathrm{O}$ strcching): EIMS. $\mathrm{m} / \%(\mathrm{rcl}$. intensily) 194 (M . 1). 179 (48). 177 (3). $167(8) .149$ (18).

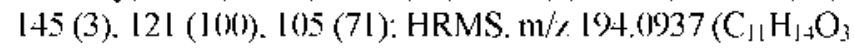
requires $194,(09+3)$.

38a: 'H NMR 0.22 (s. 9H. OSiMc) $) .77$ (s. 3H. C(Ph)$\mathrm{CH}_{3}$ ). 2.74 (s. $3 \mathrm{H}, \mathrm{OCH}_{3}$ ). 4.60 (d. $1 \mathrm{H} . J=6.7 \mathrm{~Hz} .-\mathrm{OCH}_{2}$ ). 4.66 (d. $\left.1 \mathrm{H}, J=6.6 \mathrm{~Hz} .-\mathrm{OCH}_{2}\right), 7.31-7.54$ (In. $5 \mathrm{H}$, aromatic): ${ }^{1 .} \mathrm{C}$ NMR $1.1\left(\mathrm{OSiMe}_{3}\right) .23 .4\left(\mathrm{C}(\mathrm{Ph}) \mathrm{CH}_{3}\right) .49 .9\left(\mathrm{OCH}_{3}\right)$. $79.0\left(\mathrm{OCH}_{2}\right), 96.1\left(\mathrm{C}(\mathrm{Ph}) \mathrm{CH}_{3}\right), 10 \mathrm{l} .6\left(\mathrm{C}\left(\mathrm{OSSMe}_{3}\right) \mathrm{OCH}_{3}\right)$. 126.3. 127.2 and 127.7 (CH. aromatic). $1+1.9$ (C. aromatic): Ir (neat) $1210 \mathrm{~cm}^{-1}$ (C-O streching): EIMS. $\mathrm{nv} / \mathrm{z}$ (rel. intenlsity) 266 (0.1). 236 (39). 207 (20). 191 (32). 146 (100). 89 (29): HRMS. $266.1337\left(\mathrm{C}_{4}+\mathrm{H}_{22} \mathrm{O}_{3}\right.$ Si requires 266.1338).

38b: 'H NMR -0.14 (s. 9H. OSiMe $), 1.70$ (s. 3H. C(Ph)$\mathrm{CH}_{3}$ ). 3.37 (s. $3 \mathrm{H}, \mathrm{OCH}_{3}$ ). 4.49 (d. $1 \mathrm{H} . J=6.8 \mathrm{~Hz} .-\mathrm{OCH}_{2}$ ). 4.63 (d. $\left.1 \mathrm{H}, J=6.9 \mathrm{~Hz},-\mathrm{OCH}_{2}\right), 7.22-7.38$ (m. $5 \mathrm{H}$, aromatic): ${ }^{1.9} \mathrm{C} \mathrm{NMR} 0.0\left(\mathrm{OSiMe}_{3}\right) .23 .6\left(\mathrm{C}(\mathrm{Pl}) \mathrm{CH}_{3}\right) .49 .8\left(\mathrm{OCH}_{3}\right)$. $75.7\left(\mathrm{OCH}_{2}\right), 96.7\left(\mathrm{C}\left(\mathrm{Ph}^{2} \mathrm{CH}_{3}\right), 100.1\left(\underline{\mathrm{C}}\left(\mathrm{OSiMe}_{3}\right) \mathrm{OCH}_{3}\right)\right.$. 125.0. 126.0 and $126.9(\mathrm{CH}$, aronatic), $1+2.1$ (C. aromatic): Ir (neat) $1220 \mathrm{~cm}^{-1}$ (C-O strecluing): EIMS. $\mathrm{nl} / \mathrm{z}$ (rel. intenlsity) $266\left(\mathrm{M}^{-}, 0.2\right), 251$ (2). 236 (5). 219 (1). 207 (3). 193 (12). 151 (12). 146 (100): HRMS, $266.1337\left(\mathrm{C}_{1+1} \mathrm{H}_{22} \mathrm{O}_{2} \mathrm{Si}\right.$ requires 266.1338 )

Benzene. A solution of acetophenone (2) $(430 \mathrm{mg} .3 .6$ $\mathrm{mmol}$ ) and ketene acetal 11 (1.05 g. $7.2 \mathrm{mmol}$ ) in $100 \mathrm{~mL}$ of 
benzene was irradiated for 16 h (ca. $65 \%$ conversion of 2 ). Work-up and chromatographic $(1: 4$, ethy 1 acetate $: n$ hexanc. v/s) separation (sec Gencral) gave the diastreomeric oxclanes 38a (130 mg. $21 \%$ ) and 38b (200 mg. 32\%). and $100 \mathrm{mg}(22 \%)$ or 24 .

Irradiation of Benzophenone (3) and 1-(Methoxy)-1(trimethylsilyloxy)ethane (11). Benzene. A solution of ben/ophenone (3) $(656 \mathrm{mg} .3 .6 \mathrm{mmol}$ ) and ketene acetal 11 (1.05 g. $7.2 \mathrm{mmol}$ ) in $100 \mathrm{~mL}$ of benzene was irradiated for 25 h (ca. $89 \%$ cons crsion of 3 ). Work-up and chromatographic ( $1: 10$. cthy $]$ acetate $: n$-hexanc, $v / v$ ) scparation (sce General) gave $131 \mathrm{mg}(16 \%)$ of $25.710 \mathrm{mg}(68 \%)$ of 39 and $23 \mathrm{mg}(4 \%)$ of 48 .

25: ${ }^{1} \mathrm{H}$ NMR 3.30 (s. $2 \mathrm{H}_{0} \mathrm{CH}_{2}$ ). 3.65 (s. $3 \mathrm{H} . \mathrm{CO}_{2} \mathrm{CH}_{3}$ ). $5.06(\mathrm{~s} .1 \mathrm{H} . \mathrm{OH}) \cdot 7.17-7.47\left(\mathrm{~m} . \mathrm{l}\left(\mathrm{HH}\right.\right.$. aromatic): ${ }^{13} \mathrm{C} \mathrm{NMR}$ $45.3\left(\mathrm{CH}_{2}\right) .51 .9\left(\mathrm{CO}_{2} \mathrm{CH}_{3}\right) .83 .0\left(\mathrm{C}(\mathrm{OH}) \mathrm{Ph}_{2}\right) .125 .6 .127 .1$. 127.2. 128.2 and $128.6(\mathrm{CH}$. aromatic). 144.2 and $145.9(\mathrm{C}$. aromatic). $173.2(\mathrm{C}=\mathrm{O})$ : Ir (ncal) 3600$)-3200 \mathrm{~cm}^{-1}$ (br. $\mathrm{OH}$ streching): ElMS $\mathrm{m} / \%$ (rel, intensity) $256\left(\mathrm{M}^{\prime}, 3\right) .239$ (3). $184(15) .183(100)$. $148(14) .105(71) .77(26)$ : HRMS. m/t. 256. $1100\left(\mathrm{C}_{16} \mathrm{H}_{16} \mathrm{O}_{3}\right.$ requires 256.1100$)$.

39: ${ }^{1} \mathrm{H}$ NMR $-0,05$ (s. 9H. OSiMc ). 2.97 (s. 3H. OCH ${ }_{3}$ ). 4.61 (d. $\left.1 \mathrm{H} . J=6.8 \mathrm{H} \%, \mathrm{OCH}_{2}\right) .4 .72($ d. $\mathrm{lH} . J=6.7 \mathrm{H} \%$

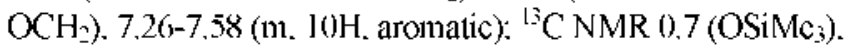
$50.0\left(\mathrm{OCH}_{3}\right) .78 .8\left(\mathrm{OCH}_{2}\right) .98 .9\left(\mathrm{C}(\mathrm{Ph})_{2}\right) .101 .7\left(\mathrm{C}\left(\mathrm{OSiMc}_{3}\right)-\right.$ $\left.\mathrm{OCH}_{3}\right), 126.8 .127 .1$ and $127.5(\mathrm{CH}$. aromatic). 141.1 (C. aromatic): Ir (ncat) $1200 \mathrm{~cm}^{-1}$ (C-O strcching): EIMS. $\mathrm{m} / \mathrm{t}$ (rel. intensity) 328 (M . 0.2). 313 (1). 269 (3). 255 (8). 193 (8). $164(12), 147$ (13). l16 (100): HRMS. m/ . 328. 1495 $\left(\mathrm{C}_{19} \mathrm{H}_{2+} \mathrm{O}_{3} \mathrm{Si}\right.$ requires 328.1495$)$.

Irradiation of 4-Cyanoacetophenone (5) and 1-(Methoxy)-1-(trimethylsilyloxy) ethane (11). Benzene. A solution of 4-cyanoacctophenone (5) $(520 \mathrm{mg} .3 .6 \mathrm{mmol})$ and kelene acctal 11 (1.05 g. $7.2 \mathrm{mmol})$ in $100 \mathrm{~mL}$ of ben/sene was irradiated for 25 h ( $\mathrm{ca} .43 \%$ conversion of 5 ). Work-up and chromatographic $(1: 5$. chlyl acclate $: n$-hexanc. $1 / y)$ scparation (sce General) gave the diastrcomeric oxctanes $40 \mathrm{a}(63 \mathrm{mg} .14 \%)$ and $40 \mathrm{~b}(144 \mathrm{mg} .32 \%) .136 \mathrm{mg}(40 \%)$ of 26 and $1+\mathrm{mg}(6 \%)$ of diol 49 .

26: ${ }^{1} \mathrm{H}$ NMR 1.53 (s. $3 \mathrm{H} . \mathrm{C}(\mathrm{OH}) \mathrm{CH}_{3}$ ). 2.82 (d. $1 \mathrm{H} . J=$ $\left.16.0 \mathrm{~Hz} . \mathrm{CH}_{2}\right) .2 .98\left(\right.$ d. $\left.1 \mathrm{H}, J=16.0 \mathrm{~Hz}, \mathrm{CH}_{2}\right) .3 .62$ (s. $3 \mathrm{H}$. $\left.\mathrm{CO}_{2} \mathrm{CH}_{3}\right), 4.46(\mathrm{~s}, 1 \mathrm{H}, \mathrm{OH}), 7.54-7.66(\mathrm{ml} .4 \mathrm{H}$, aromatic): ${ }^{1.3} \mathrm{C}$ NMR $30.3\left(\mathrm{C}(\mathrm{OH}) \mathrm{CH}_{3}\right) .45 .6\left(\mathrm{CH}_{2}\right) .51 .9\left(\mathrm{CO}_{2} \mathrm{CH}_{3}\right)$. $72.5\left(\mathrm{C}(\mathrm{OH}) \mathrm{CH}_{3}\right), 110.8(\mathrm{CN}), 125.4$ and $132.1(\mathrm{CH}$. aromatic). 118.7 and $152.2(\mathrm{C}$. aromatic). $172.6(\mathrm{C}=\mathrm{O})$ : $\operatorname{Ir}$ (neat) $3600-3200$ (br. OH streching), 2230 (CN streching). $1730 \mathrm{~cm}^{-1}(\mathrm{C}=\mathrm{O}$ streching); CIMS $\mathrm{m} / \mathrm{z}$ (rel. intensity) 220 $\left(\mathrm{M}^{+}+1,12\right), 203(50), 202(13), 172(10), 147(11) .146$ (100). 130 (83): HRMS. $\mathrm{m} / \mathrm{z} 220.0975\left(\mathrm{C}_{12} \mathrm{H}_{1+} \mathrm{NO}_{3}\right.$ requires 220.0974).

40a: 'H NMR 0.22 (s. 9H, OSiMe $), 1.72$ (s. 3H. C(Plh$\mathrm{CN}) \underline{\mathrm{H}}_{3}$ ). 2.80 (s. $3 \mathrm{H} . \mathrm{OCH}_{3}$ ). 4.58 (d. $1 \mathrm{H} . J=6.8 \mathrm{~Hz}$. $\left.\mathrm{OCH}_{2}\right) .4 .64$ (d. $\left.1 \mathrm{H} . J=6.9 \mathrm{~Hz}, \mathrm{OCH}_{2}\right) .7 .56-7.67(\mathrm{~m} .4 \mathrm{H}$. aromatic): ${ }^{1.3} \mathrm{C} \mathrm{NMR} 1.1\left(\mathrm{OSiMe}_{3}\right) .23 .5\left(\mathrm{C}(\mathrm{OH}) \mathrm{CH}_{3}\right) .50 .1$ $\left(\mathrm{OCH}_{3}\right) .78 .9\left(\mathrm{OCH}_{2}\right) .95 .8\left(\mathrm{C}\left(\mathrm{Ph}_{1}-\mathrm{CNN}_{3}\right) \mathrm{CH}_{3}\right) .101 .4\left(\underline{\mathrm{C}}\left(\mathrm{OSiMe}_{3}\right)-\right.$ $\left.\mathrm{OCH}_{3}\right) .111 .0(\mathrm{CN}), 126.9$ and $131.5(\mathrm{CH}$ aromatic) 119.0 and 147.6 (C. aromatic): Ir (neat) 2230 (CN streching). 1220 $\mathrm{cm}^{-1}$ (C-O streching): EIMS, $\mathrm{m} / \mathrm{t}$ (rcl. intensity) 291 (M'. 0.1). $266(0.3) .249$ (2). 157 (28). $146(88) .129(28) .89$ (66). 73 (100): HRMS, m/. 291.1289 $\left(\mathrm{C}_{15} \mathrm{H}_{21} \mathrm{NO}_{3}\right.$ requires 291.1291).

40b: ${ }^{1} \mathrm{H}$ NMR -0.12 (s. 9H. OSiMc $) .1 .67$ (s. 3H. C(Ph$\mathrm{CN}) \mathrm{CH}_{3}$ ). 3.36 (s. $\left.3 \mathrm{H}, \mathrm{OCH}_{3}\right) .4 .46$ (d. $1 \mathrm{H} . J=7.0 \mathrm{H} /$. $\left.\mathrm{OCH}_{2}\right) .4 .6 \mathrm{l}\left(\mathrm{d} . \mathrm{lH}, J=7,0 \mathrm{H} \% \mathrm{OCH}_{2}\right) .7,46-7,67(\mathrm{~m},+\mathrm{H}$. aromatic): ${ }^{13} \mathrm{C}$ NMR $0.6\left(\mathrm{OSiMc}_{3}\right), 24.1\left(\mathrm{C}(\mathrm{OH}) \mathrm{CH}_{3}\right), 50.4$ $\left(\mathrm{OCH}_{3}\right) .78 .5\left(\mathrm{OCH}_{2}\right), 96.9\left(\mathrm{C}\left(\mathrm{Ph}_{-} \mathrm{CN}\right) \mathrm{CH}_{3}\right) .100 .4\left(\mathrm{C}\left(\mathrm{OSiMc}_{3}\right)-\right.$ $\left.\mathrm{OCH}_{3}\right) .110 .4(\mathrm{CN}), 126.3$ and $131.4(\mathrm{CH}$, aromatic). 119.0 and 148.4 (C. aromatic): $\operatorname{lr}$ (ncat) 2230 ( $\mathrm{CN}$ streching). 1220 $\mathrm{cm}^{-1}$ (C-O streching): EIMS. $\mathrm{m} / \%$ (rcl. intensity) $291\left(\mathrm{M}^{1}\right.$. 0.3). $266(2) .247$ (1). 232 (2). 224 (1). 216 (2). 206 (1). 15 ) (62). $130(70) .102(46) .69(100)$ : HRMS. m/. 291.1288 $\left(\mathrm{C}_{15} \mathrm{H}_{21} \mathrm{NO}_{3} \mathrm{Si}\right.$ requires 291.1291).

Irradiation of Butyrophenone (6) and 1-(Methoxy)-1(trimethylsilyloxy)ethane (11). Acetonitrile. A solution of buty rophenone (6) $(500 \mathrm{mg} .3 .4 \mathrm{mmol}$ ) and ketene acetal 11 $(990 \mathrm{mg} .6,8 \mathrm{mmol})$ in $1(0) \mathrm{mL}$ of acclonitrilc was irradiated for $2 \mathrm{~h}$ ( $\mathrm{ca} .95 \%$ conversion of 6 ). Work-up and chromatographic (1 : 18. chyl acctate : $n$-hexanc. $y /$ ) separation (sec Gencral) gave the diastreomeric oxctanes 31 a $(27 \mathrm{mg} .3 \%)$ and $31 \mathrm{~b}(60 \mathrm{mg}$. $7 \%$ ). $244 \mathrm{mg}(63 \%)$ of 2 and diol $80 \mathrm{mg}$ (17\%) of 50).

Irradiation of Acetophenone (2) and 3,4-Dihydro-6(trimethylsilyloxy)-2H-pyranc (12). Acetonitrile. A solution of acclophenone (2) (360 mg. $3.0 \mathrm{mmol}$ ) and ketenc acctal $12(1.03 \mathrm{~g} .6 .0 \mathrm{mmol})$ in $100 \mathrm{~mL}$ of acctonitrile was irradiated for 37 h ( $c a .30 \%$ conversion of 2 ). Work-up and chromatographic ( $1: 4$. cthyl acctatc : $n$-hexanc. $v / v)$ separation (sce General) gave $42 \mathrm{mg}(21 \%)$ of 4 .

44: ${ }^{1} \mathrm{H}$ NMR 1,64-1.74 (m. 2H. $\mathrm{CH}_{2} \mathrm{CH}_{2} \mathrm{CH}_{2}$ ). 1.78 (s. $3 \mathrm{H}$. $\left.\mathrm{CH}_{3}\right) .1 .78-1.82\left(\mathrm{~m} .2 \mathrm{H} . \mathrm{CHC} \mathrm{H}_{2}\right) .2 .90(\mathrm{t} . \mathrm{lH} . J=10.0 \mathrm{H} \%$ $\mathrm{CHCH}_{2} \mathrm{CH}_{2}$ ). $4.1 \mathrm{l}-4.32$ (m. $2 \mathrm{H} . \mathrm{CO}_{2} \mathrm{CH}_{2}$ ). 4.45 (s. $1 \mathrm{H}$. $\mathrm{OH}) .7 .26-7.46\left(\mathrm{~m} .5 \mathrm{H}\right.$. aromatic): ${ }^{13} \mathrm{C}$ NMR $21.0\left(\mathrm{CH}_{2} \mathrm{CH}_{2}-\right.$ $\left.\mathrm{CH}_{2}\right) .22 .1\left(\mathrm{CHCH}_{2}\right) .29 .4\left(\mathrm{C}(\mathrm{OH}) \mathrm{CH}_{3}\right) .49 .3\left(\mathrm{CHCH}_{2} \mathrm{CH}_{2}-\right)$. $68.1\left(\mathrm{CO}_{2} \mathrm{CH}_{2}-\right) .74 .5\left(\mathrm{C}(\mathrm{OH}) \mathrm{CH}_{3}\right) .125 .1$. 126.8 and 128.2 (C.H. aromatic), $144.8(\mathrm{C}$, aromatic), $174.9(\mathrm{C}=\mathrm{O}): \operatorname{Ir}$ (neat) $3600-3000$ (br. OH streching), $1720 \mathrm{~cm}^{-1}$ ( $\mathrm{C}=\mathrm{O}$ streching): EIMS. $\mathrm{m} / \mathrm{z}$ (rel. intensity) $220\left(\mathrm{M}^{-} .0 .3\right) .205$ (1). $1+6(2)$. 131 (2). 121 (51). $120(23) \cdot 115(2) .106(8) .104$ (100). 77 (55): HRMS. $\mathrm{m} / \mathrm{z} 220.1096\left(\mathrm{C}_{13} \mathrm{H}_{16} \mathrm{O}_{3}\right.$ requires 220.1099$)$.

Irradiation of Benzophenone (3) and 3,4-Dihydro-6(trimethylsilyloxy)-2H-pyrane (12). Acetonitrile. A solution of benzophenone (3) $(547 \mathrm{mg} .3 .0 \mathrm{mmnol})$ and ketene acetal $12(1.03$ g. $6.0 \mathrm{~mm}$ mol) in $100 \mathrm{~mL}$ of acetonitrile was irradiated for 21 h (ca. $61 \%$ conversion of 3 ). Work-up and chromatographic $(1: 10$. etlyl acetate $: n$-hexane, $v / v)$ separation (see General) gave $103 \mathrm{mlg}(20 \%)$ of $\mathbf{4 5}$ and $67 \mathrm{mg}$ $(20 \%)$ of 48 .

45: $\mathrm{mp} 150-152^{\circ} \mathrm{C}:{ }^{1} \mathrm{H}$ NMR $1.45-1.58$ (m. $2 \mathrm{H} . \mathrm{CH}_{2} \mathrm{C}_{2}-$ $\mathrm{CH}_{2}$ ). $1.81-1.97$ (m. $2 \mathrm{H}, \mathrm{CHC} \underline{\mathrm{H}}$ ) .3 .62 (dd. $1 \mathrm{H} . J=8.0 \mathrm{~Hz}$ and $J=12.0 \mathrm{~Hz}, \mathrm{CH}) .4 .38-4.50$ (1m. 2H, $\left.\mathrm{CO}_{2} \mathrm{CH}_{-}-\right) .4 .52$ (s. $1 \mathrm{H} . \mathrm{OH}), 7.17-7.46\left(\mathrm{~m}, 10 \mathrm{H}\right.$. aromatic): ${ }^{1.3} \mathrm{C}$ NMR 20.5 $\left(\mathrm{CH}_{2} \mathrm{CH}_{2} \mathrm{CH}_{2}\right) .22 .1\left(\mathrm{CHCH}_{2}\right), 46.8\left(\mathrm{CO}_{2} \mathrm{CH}_{2}-\right) .67 .8(\underline{\mathrm{CH}}-$ $\left.\mathrm{CH}_{2} \mathrm{CH}_{-}-\right) .77 .8\left(\mathrm{C}(\mathrm{OH}) \mathrm{Ph}_{2}\right), 125.7,125.8 .126 .7 .127 .0$. 128.1 and $128.2(\mathrm{CH}$, aromatic). 144.0 and 146.7 (C. 
aromatic). $175,2(\mathrm{C}=\mathrm{O}): \operatorname{lr}(\mathrm{KBr}) 3200-3600$ (br $\mathrm{OH}$ streching). $1710 \mathrm{~cm}^{-1}(\mathrm{C}=\mathrm{O}$ streching): ElMS, $\mathrm{m} / \mathrm{s}$ ( $(\mathrm{rel}$. intensity) $282\left(\mathrm{M}^{\prime}, 2\right), 265(22), 205(6), 185$ (44), 183 (100). 165 (5). 105 (18). 104 (97): HRMS. m/. 282.1251 $\left(\mathrm{C}_{18} \mathrm{H}_{18} \mathrm{O}_{3}\right.$ requires 282.1256$)$.

lrratiation of 4-Cyanoacetophenone (5) and 3,4-Dihydro-6-(trimethỵlsilyloxy)-2H-pyrane (12). Benzene. A solution of 4 -cyanoacetophenone (5) $(435 \mathrm{mg} .3 .0 \mathrm{mmol}$ ) and ketene accial 12 (1.03 g. $6.0 \mathrm{mmol})$ in $100 \mathrm{~mL}$ of benzene was irradiated for 25 h ( $c a .54 \%$ conversion of 5 ). Work-up and chromatographic ( $1: 4$. ether : $n$-hexane. $/ /)$ scparation (see General) gave $80 \mathrm{mg}(20 \%)$ or 46 and $148 \mathrm{mg}$ $(50 \%)$ of 49

46: ${ }^{l} \mathrm{H}$ NMR $1.52-1.69\left(\mathrm{~m}, 2 \mathrm{H} . \mathrm{CH}_{2} \mathrm{CH}_{2} \mathrm{CH}_{2}\right), 1.77$ (s. $3 \mathrm{H}$. $\left.\mathrm{C}(\mathrm{OH}) \mathrm{CH}_{3}\right) .1 .80-1.88\left(\mathrm{~m} .2 \mathrm{H} . \mathrm{CHCH}_{2}\right) .2 .89(1.1 \mathrm{H} . J=9.5$ $\mathrm{H} / . \mathrm{CHCH}_{2} \mathrm{CH}_{2}$ ) $4.2 \mathrm{l}-4.28$ (n. $2 \mathrm{H}_{2} \mathrm{CO}_{2} \mathrm{CH}_{2}-$ ). 4.40) (s. l H. $\mathrm{OH}) .7 .55$ (d. $2 \mathrm{H} . J=8.5 \mathrm{H} /$. aromatic). 7.65 (d. $2 \mathrm{H} . J=8.5$ $\mathrm{H} \%$ aromatic): ${ }^{1 \mathrm{C}} \mathrm{NMR} 20.7\left(\mathrm{CH}_{2} \mathrm{CH}_{2} \mathrm{CH}_{2}\right) .21 .9\left(\mathrm{CHCH}_{2}\right)$. $29.3\left(\mathrm{C}(\mathrm{OH}) \mathrm{CH}_{3}\right) .48 .6\left(\mathrm{CHCH}_{2} \mathrm{CH}_{2}-\right) .68 .0\left(\mathrm{CO}_{2} \mathrm{CH}_{2}-\right) .74 .4$ $\left(\mathrm{C}(\mathrm{OH}) \mathrm{CH}_{3}\right) .110 .9(\mathrm{CN}) .125 .5$ and $131.9(\mathrm{CH}$. aromatic $)$. 118.6 and $150.3(\mathrm{C}$. aromatic). $176,6(\mathrm{C}=\mathrm{O})$ : Ir (ncat) 3600 3200 (br. OH streching). 2240 (CN streching). $1740 \mathrm{~cm}^{-1}$ ( $\mathrm{C}=\mathrm{O}$ streching): $\mathrm{CIMS} . \mathrm{m} / \%$ (rcl, intensity) $246\left(\mathrm{M}^{\prime}+\mathrm{l} . \mathrm{l}\right)$. 231 (2). $230(16) .186(1) .146(25) .130(65) .116(3) .103$ (4). $102(22) .100(100)$ : HRMS. $\mathrm{m} / . .246 .1131\left(\mathrm{C}_{14} \mathrm{H}_{16} \mathrm{NO}_{3}\right.$ requires 246.1130 ).

Acknowledgment. This research was supported by grants from the Basic Rescarch Program of Korca Science and Engineering Foundation (2000)-1-123-(0)6-3) and Basic Rcscarch Institute Program. Ministry of Education of Korca (BSRI-95-and-96-3408). PSM acknow ledges linancial support from NIH and NSF.

\section{References}

1. (a) Yoon. U. C.: Mariano. P. S. lece (hem. Res. 1992, 25. 233. (b) Yoon. U. C.: Mariano. P. S. Icc. Chtm. Res. 2001, 34,523.

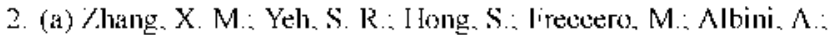
Jalver 1). li. Mariano, P. S. J. Am ( Chem Soc 1994, 116, 4211 . (b) Su. Z.: Falver: D. F.: Yoon. U. C.: Oh. S. W.: Mariano. I. S. $J$ Am Chem Soc 1998. 120.10676.

3. (a) Brumfield. M. A.: Quillen. S. L.: Yoon. U. C.: Mariano. P. S. $d$. Am. Chem. Soc 1984, 106, 6855. (b) Ohga, K: Yoon, II. C.:
Mariano. P. S. J. Org (Them. 1984. 19.213.

4. IBrumlield. M. A.: Yoon, U. C.: Hasegana, L.: Mariano. P. S. J. Org them 1988, 53. 5435.

5. (a) Yoon. Y. C.: Kim. J. U.: Hasegawa, .:. Mariano. P. S. J. Am. (them. Soc: 1987. 109. 4421. (b) Hasegawa. E.: Wu. X.: Mariano. P. S.: Yoon. U. C.: Kim. T. U. J. Am. Chm. Soc, 1988. H0. 8099.

6. Yoon. U. C.: Kim. Y. C.: Choi. T. T.: Kim. D. U.: Mariano. P. S.: Cho. 1. S.: Jeon. Y. T. J. Org. Chom 1992, 57, 1422

7. (a) Yoon U. C. Kim. II. I. Mariano. P. S. Hetorocleles 1989. 29. Io41: (b) Yoon. U. C. Cho. S. I. Oh. J. II. I ee, J. G.: Kang. K. T.: Mariano. P. S. Bull. Konem (hem. Soc. 1991. 12. 241. (c) Yoon. U. C.: Oh. T. H.: Lec. S. J.: Kim. D. U.: Lec. J. G.: Kang. K. T.: Mariano. P. S. Bull Komeon (Then. Soc. 1992. 13. 166. (d) Yoon. U. C . I ee. S. I.: I ee. K J.: Cho. S. I. I ee C W W Mariano. P. S. Bull . Sorean (7hem. Soc. 1994, 15(2) 154 (e) Yoon. U. C: Oh. S. W. I dee. C. W. Itenocyctes 1995, H(2), 2665. (1) Yoon. U. C.: Kim. J. W.: Rvut. J. Y.: Cho. S. T.: Oh. S. W.: Mariano. P. S. J. Photochem. Photobiol. A 1997. 106. 145. (g) Yoon. U. C.: Oh. S. W.: Lee. J. H.: Park. J. H.: Kang. K. T.: Mariano. P. S. J. Org. Chem. 2001, 66, 939 .

8. (a) Amold 1). R. idwances in Photochemistry. Noves. W. A. Ilammond: Pitts, J. N.. lids: Interscience Publishers: New York. 1968: Vol, 6. (b) Tones. G. Orgamic Photochemismy: Padwa. A.. Ed.: Marcel Dekker: New York. 1981: Vol. 5. Chapter 1.

9. Results reported in this paper were taken from the master work of M. J. I. al the Pusan National University (Ieburary 1992) and were presented at the I6th IUPAC Symposium on Photochemistry. July 1996 (Abstract pp. 6(N) I I lelsink, limland, the lst KoreanFrench Seminar on Organic Chemistry. November 1995 (Abstract pp. 190). Scoul. Korea and Japan-Korea Seminar on Syrnthetic Organic Chemistry for Young Chemists. November 1995 (A bstract pp. 7), Kagoshima. Japan.

10. (a) Regioselective formation of 2-alkoxyoxetanes in the photoreaction of aromalic carbonyl compound $\mathrm{w}$ ith 9 and the ellect of solvent and silyl group have been reported ${ }^{\text {lob }}$ (b) Abe. M.: Shirodai. Y.: Nojima. M. J. Chem. Soc: Puhin Troms. 1998. 3253. (c) First example of photoaddition reaction of silyl ketenc acelal to aromatic carbonyl compound was reported by

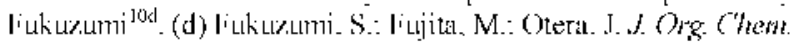
1993. $58,5415$.

11. Murow. S. L.: Carmichacl. I.: Hug. G. L. Hundtook of Photochemisty. 2nd Ed.: Mareel Dekker: New York. 1993.

12. (a) Renaud. R. N.: Berube. D.: Stephens. C. T. C'an. J. Chem. 1983. 6I. 1.37\%. (b) Corrected for $\Lambda \mathrm{g} 0.01 \mathrm{M} \wedge \mathrm{gNO}, 0.1 \mathrm{M}$ lic.10, in acetonitrile.

13. Manrr. C. K.: I3ames. K. K. Electrochemical Reactions in Nonaquons Systms: Marcel Dekker: New York. 1970: p 184.

14. Rehm. D.: Weller. A. Ist J. (Them. 1970. 8. 259.

15. (a) Wagner. P. J. icc. Chem. Res 1971. t. 169. (b) Wagcr. P. J.: I lammond. G. S. f. im Chem. Soc. 1966, 88. 1245. 